\title{
Mildly Inertial Subgradient Extragradient Method for Variational Inequalities Involving an Asymptotically Nonexpansive and Finitely Many Nonexpansive Mappings
}

\author{
Lu-Chuan Ceng ${ }^{1}$, Xiaolong Qin ${ }^{2}$, Yekini Shehu ${ }^{3}$ and Jen-Chih Yao ${ }^{4, *}$ \\ 1 Department of Mathematics, Shanghai Normal University, Shanghai 200234, China; zenglc@shnu.edu.cn \\ 2 General Education Center, National Yunlin University of Science and Technology, Douliou 64002, Taiwan; \\ qinxl@yuntech.edu.tw \\ 3 Department of Mathematics, Zhejiang Normal University, Jinhua 321004, China; yekini.shehu@unn.edu.ng \\ 4 Research Center for Interneural Computing, China Medical University Hospital, Taichung 40447, Taiwan \\ * Correspondence: yaojc@mail.cmu.edu.tw
}

Received: 13 July 2019; Accepted: 19 September 2019; Published: 22 September 2019

check for updates

\begin{abstract}
In a real Hilbert space, let the notation VIP indicate a variational inequality problem for a Lipschitzian, pseudomonotone operator, and let CFPP denote a common fixed-point problem of an asymptotically nonexpansive mapping and finitely many nonexpansive mappings. This paper introduces mildly inertial algorithms with linesearch process for finding a common solution of the VIP and the CFPP by using a subgradient approach. These fully absorb hybrid steepest-descent ideas, viscosity iteration ideas, and composite Mann-type iterative ideas. With suitable conditions on real parameters, it is shown that the sequences generated our algorithms converge to a common solution in norm, which is a unique solution of a hierarchical variational inequality (HVI).
\end{abstract}

Keywords: inertial subgradient extragradient method; pseudomonotone variational inequality; asymptotically nonexpansive mapping; sequentially weak continuity

\section{Introduction}

Let $C$ be a convex and closed nonempty set in a real Hilbert space $(H,\|\cdot\|)$ with inner product $\langle\cdot, \cdot\rangle$. Let $\operatorname{Fix}(S)$ indicate the fixed-point set of a non-self operator $S: C \rightarrow H$, i.e., $\operatorname{Fix}(S)=\{u \in$ $C: u=S u\}$. One says that a self operator $T: C \rightarrow C$ is asymptotically nonexpansive if and only if $\left\|T^{n} u-T^{n} v\right\| \leq\left(1+\theta_{n}\right)\|u-v\| \forall n \geq 1, u, v \in C$, where $\lim _{n \rightarrow \infty} \theta_{n}=0$ is a real sequence. In the case of $\theta_{n}=0 \forall n \geq 1$, one says that $T$ is nonexpansive. Both the class of nonexpansive operators and asymptotically nonexpansive operators via various iterative techniques have been studied recently; see, e.g., the works by the authors of [1-13]. Let $A: H \rightarrow H$ be a self operator. Consider the classical variational inequality problem (VIP) of consisting of $u^{*} \in C$ such that

$$
\left\langle A u^{*}, v-u^{*}\right\rangle \geq 0 \forall v \in C .
$$

The set of solutions of problem (1) is indicated by $\operatorname{VI}(C, A)$. Recently, many authors studied the VIP via mean-valued and projection-based methods; see, e.g., the works by the authors of [14-21]. In 1976, Korpelevich [22] first designed and investigated an extragradient method for a solution of problem (1), that is, for arbitrarily given $u_{0} \in C,\left\{u_{n}\right\}$ is the sequence constructed by

$$
\left\{\begin{array}{l}
v_{n}=P_{C}\left(u_{n}-\tau A u_{n}\right) \\
u_{n+1}=P_{C}\left(u_{n}-\tau A v_{n}\right) \quad \forall n \geq 0
\end{array}\right.
$$


with $\tau \in\left(0, \frac{1}{L}\right)$. If problem (1) has a solution, then he showed the weak convergence of $\left\{u_{n}\right\}$ constructed by (2) to a solution of problem (1). Since then, Korpelevich's extragradient method and its variants have been paid great attention to by many scholars, who improved it in various techniques and approaches; see, e.g., the works by the authors of [23-34].

Let $\left\{T_{i}\right\}_{i=1}^{N}$ be $N$ nonexpansive mappings on $H$, such that $\Omega=\cap_{i=1}^{N} \operatorname{Fix}\left(T_{i}\right) \neq \varnothing$. Let $F$ be a $\kappa$-Lipschitzian, $\eta$-strongly monotone self-mapping on $H$, and $f$ be a contractive map with constant $\delta \in(0,1)$. In 2015, Bnouhachem et al. [2] introduced an iterative algorithm for solving a hierarchical fixed point problem (HFPP) for a finite pool $\left\{T_{i}\right\}_{i=1}^{N}$, i.e., for arbitrarily given $x_{0} \in H$, the sequence $\left\{x_{n}\right\}$ is constructed by

$$
\left\{\begin{array}{l}
y_{n}=\left(1-\beta_{n}\right) T_{N, n} T_{N-1, n} \cdots T_{1, n} x_{n}+\beta_{n} x_{n} \\
x_{n+1}=\gamma_{n} x_{n}+\left(\left(1-\gamma_{n}\right) I-\alpha_{n} \mu F\right) y_{n}+\alpha_{n} \rho f\left(y_{n}\right), \quad \forall n \geq 0
\end{array}\right.
$$

where $T_{i, n}=\left(1-\delta_{i, n}\right) I+\delta_{i, n} T_{i}$ and $\delta_{i, n} \in(0,1)$ for integer $i \in\{1,2, \ldots, N\}$. Let the parameters satisfy $0<\mu \kappa^{2}<2 \eta$ and $0 \leq \rho \tau<v$, with $v=\mu\left(\eta-\frac{\mu \kappa^{2}}{2}\right)$. Also, suppose that the sequences $\left\{\alpha_{n}\right\},\left\{\beta_{n}\right\},\left\{\gamma_{n}\right\} \subset(0,1)$ satisfy the following requirements.

(i) $\sum_{n=0}^{\infty} \alpha_{n}=\infty$ and $\lim _{n \rightarrow \infty} \alpha_{n}=0$;

(ii) $\left\{\beta_{n}\right\} \subset[\sigma, 1)$ and $\lim _{n \rightarrow \infty} \beta_{n}=\beta<1$;

(iii) $\limsup _{n \rightarrow \infty} \gamma_{n}<1$ and $\liminf _{n \rightarrow \infty} \gamma_{n}>0$;

(iv) $\lim _{n \rightarrow \infty}\left|\delta_{i, n-1}-\delta_{i, n}\right|=0$ for $i=1,2, \ldots, N$.

They proved the strong convergence of $\left\{x_{n}\right\}$ to a point $x^{*} \in \Omega$, which is only a solution to the HFPP: $\left\langle(\mu F-\rho f) x^{*}, y-x^{*}\right\rangle \geq 0 \forall y \in \Omega$.

On the other hand, let the mappings $A_{1}, A_{2}: C \rightarrow H$ be both inverse-strongly monotone and the mapping $T: C \rightarrow C$ be asymptotically nonexpansive one with $\left\{\theta_{n}\right\}$. In 2018, by the modified extragradient method, Cai et al. [35] designed a viscosity implicit method for computing a point in the common solution set $\Omega$ of the VIPs for $A_{1}$ and $A_{2}$ and the FPP of $T$, i.e., for arbitrarily given $x_{1} \in C$, the sequence $\left\{x_{n}\right\}$ is constructed by

$$
\left\{\begin{array}{l}
v_{n}=t_{n} x_{n}+\left(1-t_{n}\right) u_{n} \\
z_{n}=P_{C}\left(v_{n}-\mu A_{2} v_{n}\right) \\
u_{n}=P_{C}\left(z_{n}-\lambda A_{1} z_{n}\right) \\
x_{n+1}=P_{C}\left[\left(I-\alpha_{n} \rho F\right) T^{n} u_{n}+\alpha_{n} f\left(x_{n}\right)\right]
\end{array}\right.
$$

where $f: C \rightarrow C$ be a $\delta$-contraction with $0 \leq \delta<1$, and $\left\{\alpha_{n}\right\},\left\{t_{n}\right\}$ are the sequences in $(0,1]$ satisfying

(i) $\sum_{n=1}^{\infty} \alpha_{n}=\infty, \lim _{n \rightarrow \infty} \alpha_{n}=0$ and $\sum_{n=1}^{\infty}\left|\alpha_{n+1}-\alpha_{n}\right|<\infty$;

(ii) $\lim _{n \rightarrow \infty} \frac{\theta_{n}}{\alpha_{n}}=0$;

(iii) $0<\epsilon \leq t_{n} \leq 1$ and $\sum_{n=1}^{\infty}\left|t_{n+1}-t_{n}\right|<\infty$;

(iv) $\sum_{n=1}^{\infty}\left\|T^{n+1} u_{n}-T^{n} u_{n}\right\|<\infty$.

They proved that $\left\{x_{n}\right\}$ converges strongly to a point $x^{*} \in \Omega$, which is a unique solution to the VIP: $\left\langle(f-\rho F) x^{*}, y-x^{*}\right\rangle \leq 0 \forall y \in \Omega$.

Under the setting of extragradient approaches, we must calculate metric projections twice for every iteration. Without doubt, if $C$ is a general convex and closed subset, the computation of the projection onto $C$ might be prohibitively consuming-time. In 2011, motivated by Korpelevich's extragradient method, Censor et al. [5] first purposed the subgradient extragradient method, where a projection onto a half-space is used in place of the second projection onto $C$ :

$$
\left\{\begin{array}{l}
v_{n}=P_{C}\left(u_{n}-\ell A u_{n}\right), \\
C_{n}=\left\{u \in H:\left\langle u_{n}-\ell A u_{n}-v_{n}, u-v_{n}\right\rangle \leq 0\right\}, \\
u_{n+1}=P_{C_{n}}\left(u_{n}-\ell A v_{n}\right) \quad \forall n \geq 0,
\end{array}\right.
$$


with $\ell \in\left(0, \frac{1}{L}\right)$. In 2014, Kraikaew and Saejung [36] introduced the Halpern subgradient extragradient method for solving VIP (1) and proved that the sequence generated by the proposed method converges strongly to a solution of VIP (1).

In 2018, by virtue of the inertial technique, Thong and Hieu [37] first introduced the inertial subgradient extragradient method and proved weak convergence of the proposed method to a solution of VIP (1). Very recently, Thong and Hieu [37] introduced two inertial subgradient extragradient algorithms with the linesearch process to solve the VIP (1) for Lipschitzian, monotone operator $A$, and the FPP of quasi-nonexpansive operator $S$ satisfying the demiclosedness in $H$.

Under mild assumptions, they proved that the sequences defined by the above algorithms converge to a point in $\operatorname{Fix}(S) \cap \operatorname{VI}(C, A)$ with the aid of dual spaces. Being motivated by the research work $[2,37,38]$ and using the subgradient extragradient technique, this paper designs two mildly inertial algorithms with linesearch process to solve the VIP (1) for Lipschitzian, pseudomonotone operator, and the CFPP of an asymptotically nonexpansive mapping and finitely many nonexpansive mappings in $H$. Our algorithms fully absorb inertial subgradient extragradient approaches with linesearch process, hybrid steepest-descent algorithms, viscosity iteration techniques, and composite Mann-type iterative methods. Under suitable conditions, it is shown that the sequences constructed by our algorithms converge to a common solution of the VIP and CFPP in norm, which is only a solution of a hierarchical variational inequality (HVI). Finally, we apply our main theorems to deal with the VIP and CFPP in an illustrating example.

The outline of the article is arranged as follows. In Section 2, some concepts and preliminary conclusions are recalled for later use. In Section 3, the convergence criteria of the suggested algorithms are established. In Section 4, our main theorems are used to deal with the VIP and CFPP in an illustrating example. As our algorithms concern solving VIP (1) with Lipschitzian, pseudomonotone operator, and the CFPP of an asymptotically nonexpansive mapping and finitely many nonexpansive mappings, they are more advantageous and more subtle than Algorithms 1 and 2 in [37]. Our theorems strengthen and generalize the corresponding results announced in Bnouhachem et al. [2], Cai et al. [35], Kraikaew and Saejung [36], and Thong and Hieu [37,38].
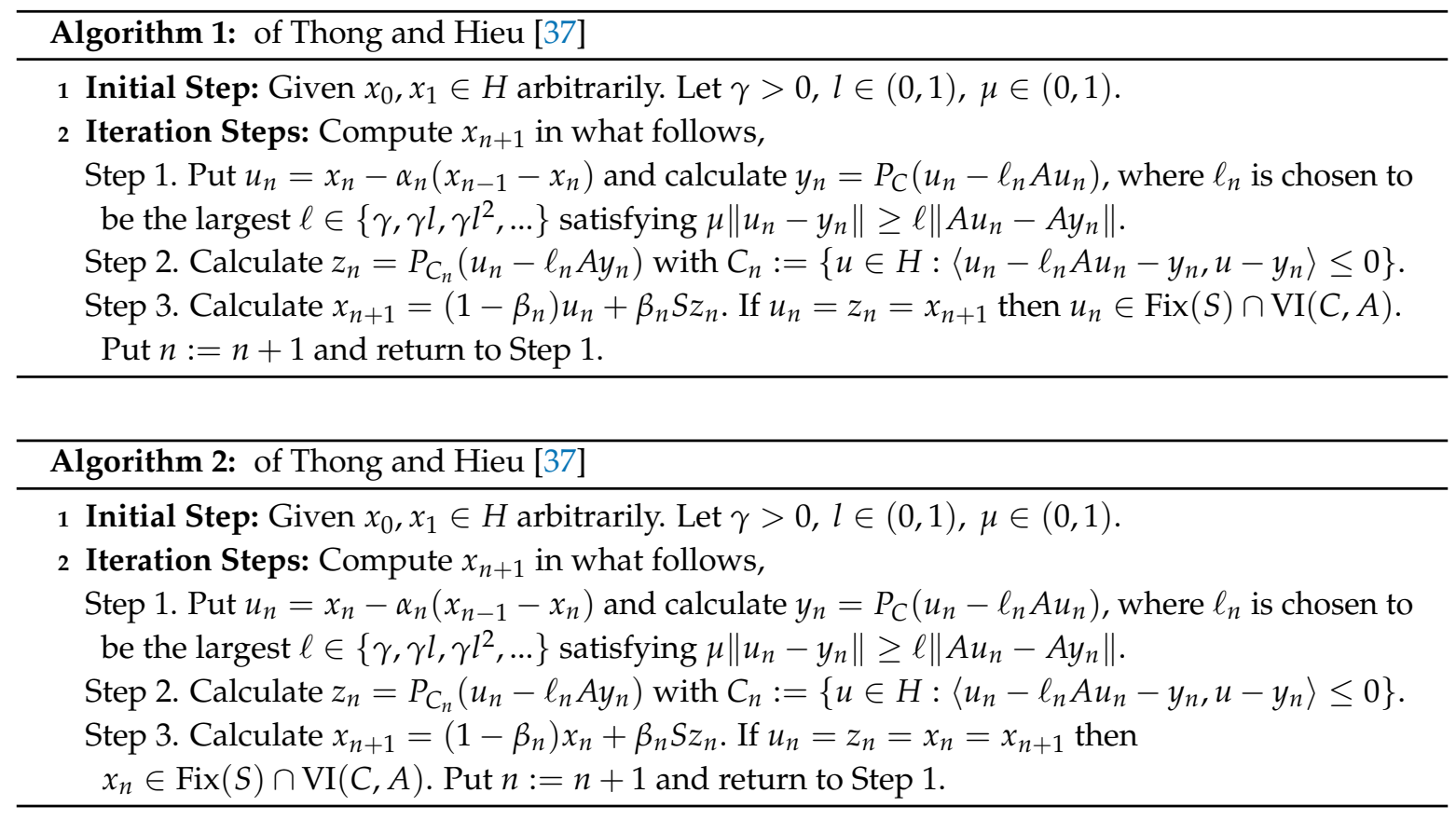


\section{Preliminaries}

Given a sequence $\left\{u_{n}\right\}$ in $H$. We use the notations $u_{n} \rightarrow u$ and $u_{n} \rightarrow u$ to indicate the strong convergence of $\left\{u_{n}\right\}$ to $u$ and weak convergence of $\left\{u_{n}\right\}$ to $u$, respectively. An operator $T: C \rightarrow H$ is said to be

(i) $L$-Lipschitz continuous (or $L$-Lipschitzian) iff $\exists L>0$ s.t.

$$
\|T u-T v\| \leq L\|u-v\| \forall u, v \in C ;
$$

(ii) monotone iff

$$
\langle T u-T v, u-v\rangle \geq 0 \forall u, v \in C
$$

(iii) pseudomonotone iff

$$
\langle T u, v-u\rangle \geq 0 \Rightarrow\langle T v, v-u\rangle \geq 0 \forall u, v \in C ;
$$

(iv) $\beta$-strongly monotone if $\exists \beta>0$ s.t.

$$
\langle T u-T v, u-v\rangle \geq \beta\|u-v\|^{2} \forall u, v \in C ;
$$

(v) sequentially weakly continuous if $\forall\left\{u_{n}\right\} \subset C$, the relation holds: $u_{n} \rightarrow u \Rightarrow T u_{n} \rightarrow T u$.

It is clear that every monotone mapping is pseudomonotone but the converse is not valid; e.g., take $T x:=\frac{a}{a+x}, \quad x, a \in(0,+\infty)$.

For every $u \in H$, we know that there is only a nearest point in $C$, indicated by $P_{C} u$, s.t. $\left\|u-P_{C} u\right\| \leq$ $\|u-v\| \forall v \in C$. The operator $P_{C}$ is said to be the metric projection from $H$ to $C$.

Proposition 1. The following hold in real Hilbert spaces:

(i) $\left\langle u-v, P_{C} u-P_{C} v\right\rangle \geq\left\|P_{C} u-P_{C} v\right\|^{2} \forall u, v \in H$;

(ii) $\left\langle u-P_{C} u, v-P_{C} u\right\rangle \leq 0 \quad \forall u \in H, v \in C$;

(iii) $\|u-v\|^{2}-\left\|u-P_{C} u\right\|^{2} \geq\left\|v-P_{C} u\right\|^{2} \forall u \in H, v \in C$;

(iv) $\|u-v\|^{2}+2\langle u-v, v\rangle=\|u\|^{2}-\|v\|^{2} \forall u, v \in H$;

(v) $\|\lambda u+(1-\lambda) v\|^{2}+\lambda(1-\lambda)\|u-v\|^{2}=\lambda\|u\|^{2}+(1-\lambda)\|v\|^{2} \forall u, v \in H, \lambda \in[0,1]$.

An operator $S: H \rightarrow H$ is called an averaged one if $\exists \alpha \in(0,1)$ s.t. $S=(1-\alpha) I+\alpha T$, where $I$ is the identity operator of $H$ and $T: H \rightarrow H$ is a nonexpansive operator. In this case, $S$ is also called $\alpha$-averaged. It is clear that the averaged operator $S$ is also nonexpansive and $\operatorname{Fix}(S)=\operatorname{Fix}(T)$.

Lemma 1. [2] If the mappings $\left\{T_{i}\right\}_{i=1}^{N}$ defined on $H$ are averaged and have a common fixed point, then $\cap_{i=1}^{N} \operatorname{Fix}\left(T_{i}\right)=\operatorname{Fix}\left(T_{1} T_{2} \cdots T_{N}\right)$.

The next result immediately follows from the subdifferential inequality of the function $\|\cdot\|^{2} / 2$.

Lemma 2. The following inequality holds,

$$
\|u+v\|^{2}-\|u\|^{2} \leq 2\langle v, u+v\rangle \quad \forall u, v \in H
$$

Lemma 3. [39] Assume that the mapping $A$ is pseudomonotone and continuous on $C$. Given a point $u \in C$. Then the relation holds: $\langle A u, v-u\rangle \geq 0 \forall v \in C \Leftrightarrow\langle A v, v-u\rangle \geq 0 \forall v \in C$. 
Lemma 4. [40] Let $\left\{t_{n}\right\}$ be a sequence in $[0,+\infty)$ satisfying the condition $t_{n+1} \leq s_{n} b_{n}+\left(1-s_{n}\right) t_{n} \forall n \geq 1$, where $\left\{s_{n}\right\}$ and $\left\{b_{n}\right\}$ lie in $\mathbf{R}:=(-\infty, \infty)$ s.t. (a) $\left\{s_{n}\right\} \subset[0,1]$ and $\sum_{n=1}^{\infty} s_{n}=\infty$, and (b) $\lim \sup _{n \rightarrow \infty} b_{n} \leq$ 0 or $\sum_{n=1}^{\infty}\left|s_{n} b_{n}\right|<\infty$. Then $t_{n} \rightarrow 0$ as $n \rightarrow \infty$.

Definition 1. An operator $S: C \rightarrow H$ is called $\zeta$-strictly pseudocontractive iff $\exists \zeta \in[0,1)$ s.t. $\|S u-S v\|^{2}-$ $\zeta\|(I-S) u-(I-S) v\|^{2} \leq\|u-v\|^{2} \forall u, v \in C$.

Lemma 5. [41] Assume that $S: C \rightarrow H$ is $\zeta$-strictly pseudocontractive. Define $T: C \rightarrow H$ by $T u=$ $\mu S u+(1-\mu) u \forall u \in C$. If $\mu \in[\zeta, 1), T$ is nonexpansive such that $\operatorname{Fix}(T)=\operatorname{Fix}(S)$.

Lemma 6. [42] Let $\ell \in(0,1], S: C \rightarrow H$ be nonexpansive, and $S^{\ell}: C \rightarrow H$ be defined as $S^{\ell} u:=$ $S u-\ell \mu F(S u) \forall u \in C$, where $F$ is $\kappa$-Lipschitzian and $\eta$-strongly monotone self-mapping on $H$. Then, $S^{\ell}$ is a contractive map provided $0<\mu<\frac{2 \eta}{\kappa^{2}}$, i.e., $\left\|S^{\ell} u-S^{\ell} v\right\| \leq(1-\ell \tau)\|u-v\| \forall u, v \in C$, where $\tau=1-\sqrt{1-\mu\left(2 \eta-\mu \kappa^{2}\right)} \in(0,1]$.

Lemma 7. [43] Assume that the Banach space X admits a weakly continuous duality mapping; the subset $C \subset X$ is nonempty, convex, and closed; and the asymptotically nonexpansive mapping $S: C \rightarrow C$ has a fixed point. Then, $I-S$ is demiclosed at zero, i.e., if the sequence $\left\{u_{n}\right\} \subset C$ satisfies $u_{n} \rightarrow u \in C$ and $u_{n}-S u_{n} \rightarrow 0$, then $u \in \operatorname{Fix}(S)$.

\section{Main Results}

In this section, we always suppose the following conditions.

- $\quad T$ is an asymptotically nonexpansive operator on $H$ with $\left\{\theta_{n}\right\}$ and $\left\{T_{i}\right\}_{i=1}^{N}$ are $N$ nonexpansive operators on $H$.

- $A$ is $L$-Lipschitzian, pseudomonotone on $H$, and sequentially weakly continuous on $C$, s.t. $\Omega=$ $\cap_{i=0}^{N} \operatorname{Fix}\left(T_{i}\right) \cap \operatorname{VI}(C, A) \neq \varnothing$ with $T_{0}:=T$.

- $f$ is a contractive map on $H$ with coefficient $\delta \in[0,1)$, and $F$ is $\kappa$-Lipschitzian, $\eta$-strongly monotone on $H$.

- $v \delta<\tau:=1-\sqrt{1-\rho\left(2 \eta-\rho \kappa^{2}\right)}$ for $v \geq 0$ and $\rho \in\left(0, \frac{2 \eta}{\kappa^{2}}\right)$.

- $\quad T_{i, n}:=\left(1-\delta_{i, n}\right) I+\delta_{i, n} T_{i}$ and $\delta_{i, n} \in(0,1)$ for $i=1,2, \ldots, N$.

- $\left\{\sigma_{n}\right\} \subset[0,1]$ and $\left\{\alpha_{n}\right\},\left\{\beta_{n}\right\},\left\{\gamma_{n}\right\} \subset(0,1)$ such that

(i) $\sup _{n \geq 1} \frac{\sigma_{n}}{\alpha_{n}}<\infty$ and $\lim _{n \rightarrow \infty} \frac{\theta_{n}}{\alpha_{n}}=0$;

(ii) $\sum_{n=1}^{\infty} \alpha_{n}=\infty$ and $\lim _{n \rightarrow \infty} \alpha_{n}=0$;

(iii) $\left\{\beta_{n}\right\} \subset[\sigma, 1)$ and $\lim _{n \rightarrow \infty} \beta_{n}=\beta<1$;

(iv) $\limsup _{n \rightarrow \infty} \gamma_{n}<1, \liminf _{n \rightarrow \infty} \gamma_{n}>0$ and $\alpha_{n}+\gamma_{n} \leq 1 \forall n \geq 1$. For example, take

$$
\alpha_{n}=\frac{1}{n+1}, \sigma_{n}=\frac{1}{(n+1)^{2}}=\theta_{n}, \beta_{n}=\frac{n}{2(n+1)}, \gamma_{n}=\frac{n}{4(n+1)} .
$$

Remark 1. For Step 2 in Algorithm 3, the composite mapping $T_{N, n} T_{N-1, n} \cdots T_{1, n}$ with $T_{i, n}:=\left(1-\delta_{i, n}\right) I+$ $\delta_{i, n} T_{i}$ and $\delta_{i, n} \in(0,1)$ for $i=1,2, \ldots, N$, has the following property,

$$
\cap_{i=1}^{N} \operatorname{Fix}\left(T_{i}\right)=\cap_{i=1}^{N} \operatorname{Fix}\left(T_{i, n}\right)=\operatorname{Fix}\left(T_{N, n} T_{N-1, n} \cdots T_{1, n}\right) \quad \forall n \geq 1,
$$

due to Lemmas 1 and 5. 


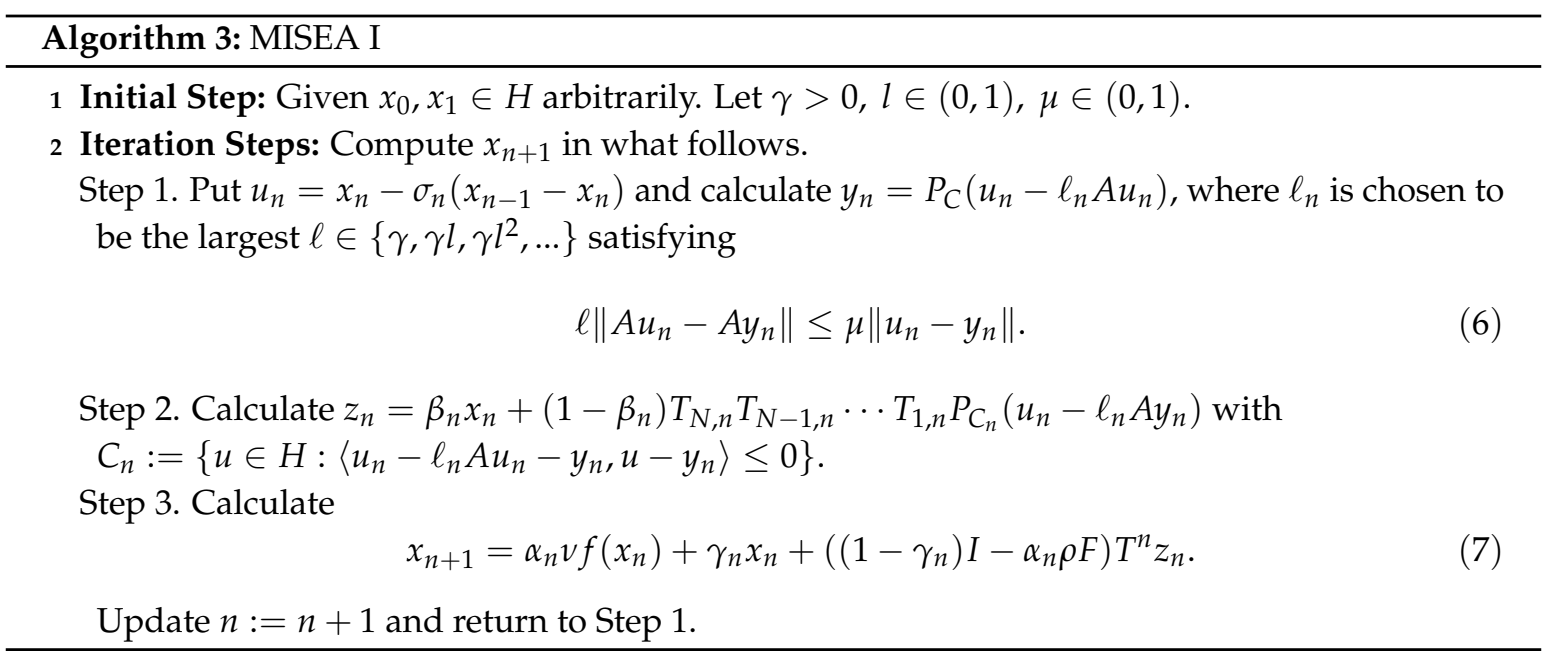

Lemma 8. The Armijo-like search rule (6) is defined well, and the following holds: $\min \left\{\gamma, \frac{\mu l}{L}\right\} \leq \ell_{n} \leq \gamma$.

Proof. As $A$ is $L$-Lipschitzian, we get $\frac{\mu}{L}\left\|A u_{n}-A P_{C}\left(u_{n}-\gamma l^{m} A u_{n}\right)\right\| \leq \mu\left\|u_{n}-P_{C}\left(u_{n}-\gamma l^{m} A u_{n}\right)\right\|$. Therefore, (6) is valid for $\gamma l^{m} \leq \frac{\mu}{L}$. This means that $\ell_{n}$ is defined well. It is clear that $\ell_{n} \leq \gamma$. In the case of $\ell_{n}=\gamma$, the inequality holds. In the case of $\ell_{n}<\gamma$, from (6) it follows that $\| A u_{n}-A P_{C}\left(u_{n}-\right.$ $\left.\frac{\ell_{n}}{l} A u_{n}\right)\left\|>\frac{\mu}{\frac{\ell_{n}}{l}}\right\| u_{n}-P_{C}\left(u_{n}-\frac{\ell_{n}}{l} A u_{n}\right) \|$. Thus, from the $L$-Lipschitzian property of $A$, we get $\ell_{n}>\frac{\mu l}{L}$. Consequently, the inequality holds.

Lemma 9. Let $\left\{u_{n}\right\},\left\{y_{n}\right\},\left\{z_{n}\right\}$ be the sequences generated by Algorithm 3. Then

$$
\begin{aligned}
\left\|z_{n}-\omega\right\|^{2} \leq & \beta_{n}\left\|x_{n}-\omega\right\|^{2}+\left(1-\beta_{n}\right)\left\|u_{n}-\omega\right\|^{2} \\
& -\left(1-\beta_{n}\right)(1-\mu)\left[\left\|u_{n}-y_{n}\right\|^{2}+\left\|v_{n}-y_{n}\right\|^{2}\right] \quad \forall \omega \in \Omega, n \geq 1,
\end{aligned}
$$

where $v_{n}:=P_{C_{n}}\left(u_{n}-\ell_{n} A y_{n}\right)$.

Proof. First, take an arbitrary $p \in \Omega \subset C \subset C_{n}$. We note that

$$
\begin{aligned}
2\left\|v_{n}-p\right\|^{2} & =2\left\|P_{C_{n}}\left(u_{n}-\ell_{n} A y_{n}\right)-P_{C_{n}} p\right\|^{2} \leq 2\left\langle v_{n}-p, u_{n}-\ell_{n} A y_{n}-p\right\rangle \\
& =\left\|v_{n}-p\right\|^{2}+\left\|u_{n}-p\right\|^{2}-\left\|v_{n}-u_{n}\right\|^{2}-2\left\langle v_{n}-p, \ell_{n} A y_{n}\right\rangle .
\end{aligned}
$$

So, it follows that $\left\|v_{n}-p\right\|^{2} \leq\left\|u_{n}-p\right\|^{2}-\left\|v_{n}-u_{n}\right\|^{2}-2\left\langle v_{n}-p, \ell_{n} A y_{n}\right\rangle$, which together with (6) and the pseudomonotonicity of $A$, deduces that $\left\langle A y_{n}, y_{n}-p\right\rangle \geq 0$ and

$$
\begin{aligned}
\left\|v_{n}-p\right\|^{2} & \leq\left\|u_{n}-p\right\|^{2}-\left\|v_{n}-u_{n}\right\|^{2}+2 \ell_{n}\left(\left\langle A y_{n}, p-y_{n}\right\rangle+\left\langle A y_{n}, y_{n}-v_{n}\right\rangle\right) \\
& \leq\left\|u_{n}-p\right\|^{2}-\left\|v_{n}-u_{n}\right\|^{2}+2 \ell_{n}\left\langle A y_{n}, y_{n}-v_{n}\right\rangle \\
& =\left\|u_{n}-p\right\|^{2}-\left\|v_{n}-y_{n}\right\|^{2}-\left\|y_{n}-u_{n}\right\|^{2}+2\left\langle u_{n}-\ell_{n} A y_{n}-y_{n}, v_{n}-y_{n}\right\rangle .
\end{aligned}
$$

As $v_{n}=P_{C_{n}}\left(u_{n}-\ell_{n} A y_{n}\right)$ with $C_{n}:=\left\{u \in H:\left\langle u_{n}-\ell_{n} A u_{n}-y_{n}, u-y_{n}\right\rangle \leq 0\right\}$, we have $\left\langle u_{n}-\right.$ $\left.\ell_{n} A u_{n}-y_{n}, v_{n}-y_{n}\right\rangle \leq 0$, which together with (6), implies that

$$
\begin{aligned}
2\left\langle u_{n}-\ell_{n} A y_{n}-y_{n}, v_{n}-y_{n}\right\rangle & =2\left\langle u_{n}-\ell_{n} A u_{n}-y_{n}, v_{n}-y_{n}\right\rangle+2 \ell_{n}\left\langle A u_{n}-A y_{n}, v_{n}-y_{n}\right\rangle \\
& \leq 2 \mu\left\|u_{n}-y_{n}\right\|\left\|v_{n}-y_{n}\right\| \leq \mu\left(\left\|u_{n}-y_{n}\right\|^{2}+\left\|v_{n}-y_{n}\right\|^{2}\right)
\end{aligned}
$$

Therefore, substituting the last inequality for (9), we obtain

$$
\left\|v_{n}-p\right\|^{2} \leq\left\|u_{n}-p\right\|^{2}-(1-\mu)\left\|u_{n}-y_{n}\right\|^{2}-(1-\mu)\left\|v_{n}-y_{n}\right\|^{2} \quad \forall p \in \Omega,
$$


which together with Algorithm 3 and $\operatorname{Fix}\left(T_{N, n} T_{N-1, n} \cdots T_{1, n}\right)=\cap_{i=1}^{N} \operatorname{Fix}\left(T_{i, n}\right)=\cap_{i=1}^{N} \operatorname{Fix}\left(T_{i}\right)$, due to Lemmas 1 and 5 implies that for all $\omega \in \Omega$,

$$
\begin{aligned}
& \left\|z_{n}-\omega\right\|^{2} \leq \beta_{n}\left\|x_{n}-\omega\right\|^{2}+\left(1-\beta_{n}\right)\left\|T_{N, n} T_{N-1, n} \cdots T_{1, n} v_{n}-\omega\right\|^{2} \\
& \leq \beta_{n}\left\|x_{n}-\omega\right\|^{2}+\left(1-\beta_{n}\right)\left\|v_{n}-\omega\right\|^{2} \\
& \leq \beta_{n}\left\|x_{n}-\omega\right\|^{2}+\left(1-\beta_{n}\right)\left[\left\|u_{n}-\omega\right\|^{2}-(1-\mu)\left\|u_{n}-y_{n}\right\|^{2}-(1-\mu)\left\|v_{n}-y_{n}\right\|^{2}\right] \\
& =\beta_{n}\left\|x_{n}-\omega\right\|^{2}+\left(1-\beta_{n}\right)\left\|u_{n}-\omega\right\|^{2}-\left(1-\beta_{n}\right)(1-\mu)\left[\left\|u_{n}-y_{n}\right\|^{2}+\left\|v_{n}-y_{n}\right\|^{2}\right] .
\end{aligned}
$$

This completes the proof.

Lemma 10. Let $\left\{u_{n}\right\},\left\{x_{n}\right\},\left\{y_{n}\right\}$, and $\left\{z_{n}\right\}$ be bounded vector sequences generated by Algorithm 3. If $T^{n} x_{n}-T^{n+1} x_{n} \rightarrow 0, x_{n}-x_{n+1} \rightarrow 0, u_{n}-y_{n} \rightarrow 0, u_{n}-z_{n} \rightarrow 0$ and $\exists\left\{w_{n_{k}}\right\} \subset\left\{u_{n}\right\}$ such that $w_{n_{k}} \rightarrow z \in H$, then $z \in \Omega$.

Proof. From Algorithm 3, we get $u_{n}-x_{n}=\sigma_{n}\left(x_{n}-x_{n-1}\right) \forall n \geq 1$, and therefore $\left\|u_{n}-x_{n}\right\|=\sigma_{n} \| x_{n}-$ $x_{n-1}\|\leq\| x_{n}-x_{n-1} \|$. Utilizing the assumption $x_{n}-x_{n+1} \rightarrow 0$, we have $u_{n}-x_{n} \rightarrow 0$. So, it follows from the assumption $u_{n}-y_{n} \rightarrow 0$, that $\left\|x_{n}-y_{n}\right\| \leq\left\|x_{n}-u_{n}\right\|+\left\|u_{n}-y_{n}\right\| \rightarrow 0(n \rightarrow \infty)$. Therefore, according to the assumption $u_{n}-z_{n} \rightarrow 0$, we get $\left\|x_{n}-z_{n}\right\| \leq\left\|x_{n}-u_{n}\right\|+\left\|u_{n}-z_{n}\right\| \rightarrow 0(n \rightarrow \infty)$. Furthermore, in terms of Lemma 9 we deduce that for each $\omega \in \Omega$,

$$
\begin{aligned}
& \left(1-\beta_{n}\right)(1-\mu)\left[\left\|u_{n}-y_{n}\right\|^{2}+\left\|v_{n}-y_{n}\right\|^{2}\right] \\
& \leq \beta_{n}\left\|x_{n}-\omega\right\|^{2}+\left(1-\beta_{n}\right)\left\|u_{n}-\omega\right\|^{2}-\left\|z_{n}-\omega\right\|^{2} \\
& \leq \beta_{n}\left\|x_{n}-\omega\right\|^{2}+\left(1-\beta_{n}\right)\left(\left\|x_{n}-\omega\right\|+\left\|x_{n}-x_{n-1}\right\|\right)^{2}-\left\|z_{n}-\omega\right\|^{2} \\
& =\beta_{n}\left\|x_{n}-\omega\right\|^{2}+\left(1-\beta_{n}\right)\left[\left\|x_{n}-\omega\right\|^{2}+\left\|x_{n}-x_{n-1}\right\|\left(2\left\|x_{n}-\omega\right\|+\left\|x_{n}-x_{n-1}\right\|\right)\right]-\left\|z_{n}-\omega\right\|^{2} \\
& =\left\|x_{n}-\omega\right\|^{2}-\left\|z_{n}-\omega\right\|^{2}+\left(1-\beta_{n}\right)\left\|x_{n}-x_{n-1}\right\|\left(2\left\|x_{n}-\omega\right\|+\left\|x_{n}-x_{n-1}\right\|\right) \\
& \leq\left(\left\|x_{n}-\omega\right\|+\left\|z_{n}-\omega\right\|\right)\left\|x_{n}-z_{n}\right\|+\left\|x_{n}-x_{n-1}\right\|\left(2\left\|x_{n}-\omega\right\|+\left\|x_{n}-x_{n-1}\right\|\right) .
\end{aligned}
$$

As $\lim _{n \rightarrow \infty}\left(1-\beta_{n}\right)=(1-\beta)>0, \mu \in(0,1), x_{n}-x_{n+1} \rightarrow 0$ and $x_{n}-z_{n} \rightarrow 0$, from the boundedness of $\left\{x_{n}\right\},\left\{z_{n}\right\}$ we get

$$
\lim _{n \rightarrow \infty}\left\|u_{n}-y_{n}\right\|=0 \quad \text { and } \quad \lim _{n \rightarrow \infty}\left\|v_{n}-y_{n}\right\|=0 .
$$

Thus we obtain that $\left\|x_{n}-v_{n}\right\| \leq\left\|x_{n}-u_{n}\right\|+\left\|u_{n}-y_{n}\right\|+\left\|y_{n}-v_{n}\right\| \rightarrow 0(n \rightarrow \infty)$.

Now, according to (7) in Algorithm 3, we have

$$
\begin{aligned}
x_{n+1}-x_{n} & =\left(1-\gamma_{n}\right)\left(T^{n} z_{n}-x_{n}\right)-\alpha_{n} \rho F T^{n} z_{n}+\alpha_{n} v f\left(x_{n}\right) \\
& =\left(1-\gamma_{n}\right)\left(T^{n} z_{n}-T^{n} x_{n}\right)+\left(1-\gamma_{n}\right)\left(T^{n} x_{n}-x_{n}\right)-\alpha_{n} \rho F T^{n} z_{n}+\alpha_{n} v f\left(x_{n}\right) .
\end{aligned}
$$

So it follows that

$$
\begin{aligned}
& \left(1-\gamma_{n}\right)\left\|T^{n} x_{n}-x_{n}\right\|=\left\|x_{n+1}-x_{n}-\alpha_{n} v f\left(x_{n}\right)-\left(1-\gamma_{n}\right)\left(T^{n} z_{n}-T^{n} x_{n}\right)+\alpha_{n} \rho F T^{n} z_{n}\right\| \\
& \leq\left\|x_{n+1}-x_{n}\right\|+\alpha_{n}\left(\left\|v f\left(x_{n}\right)\right\|+\left\|\rho F T^{n} z_{n}\right\|\right)+\left(1-\gamma_{n}\right)\left\|T^{n} z_{n}-T^{n} x_{n}\right\| \\
& \leq\left\|x_{n+1}-x_{n}\right\|+\alpha_{n}\left(\left\|v f\left(x_{n}\right)\right\|+\left\|\rho F T^{n} z_{n}\right\|\right)+\left(1+\theta_{n}\right)\left\|z_{n}-x_{n}\right\| .
\end{aligned}
$$

Since $\liminf \operatorname{in}_{n \rightarrow \infty}\left(1-\gamma_{n}\right)>0, \alpha_{n} \rightarrow 0, \theta_{n} \rightarrow 0, x_{n}-x_{n+1} \rightarrow 0$ and $x_{n}-z_{n} \rightarrow 0$, from the boundedness of $\left\{x_{n}\right\},\left\{z_{n}\right\}$ and the Lipschitz continuity of $f, F, T$, we infer that

$$
\lim _{n \rightarrow \infty}\left\|x_{n}-T^{n} x_{n}\right\|=0
$$


Also, let the mapping $W: H \rightarrow H$ be defined as $W x:=\beta x+(1-\beta) T_{N, n} T_{N-1, n} \cdots T_{1, n} x$, where $\beta \in[\sigma, 1)$. By Lemma 5 we know that $W$ is nonexpansive self-mapping on $H$ and $\operatorname{Fix}(W)=\cap_{i=1}^{N} \operatorname{Fix}\left(T_{i}\right)$. We observe that

$$
\begin{aligned}
& \left\|W x_{n}-x_{n}\right\| \leq\left\|W x_{n}-z_{n}\right\|+\left\|z_{n}-x_{n}\right\| \\
& =\left\|\left(\beta-\beta_{n}\right)\left(x_{n}-T_{N, n} T_{N-1, n} \cdots T_{1, n} x_{n}\right)+\left(1-\beta_{n}\right)\left(T_{N, n} T_{N-1, n} \cdots T_{1, n} x_{n}-T_{N}^{n} T_{N-1}^{n} \cdots T_{1}^{n} v_{n}\right)\right\| \\
& \quad+\left\|z_{n}-x_{n}\right\| \\
& \leq\left|\beta-\beta_{n}\right|\left\|x_{n}-T_{N, n} T_{N-1, n} \cdots T_{1, n} x_{n}\right\|+\left(1-\beta_{n}\right)\left\|T_{N, n} T_{N-1, n} \cdots T_{1, n} x_{n}-T_{N}^{n} T_{N-1}^{n} \cdots T_{1}^{n} v_{n}\right\| \\
& \quad+\left\|z_{n}-x_{n}\right\| \\
& \leq\left|\beta-\beta_{n}\right|\left\|x_{n}-T_{N, n} T_{N-1, n} \cdots T_{1, n} x_{n}\right\|+\left\|x_{n}-v_{n}\right\|+\left\|z_{n}-x_{n}\right\| .
\end{aligned}
$$

Since $\left\{x_{n}\right\}$ is bounded and the composite $T_{N, n} T_{N-1, n} \cdots T_{1, n}$ is nonexpansive, from $\lim _{n \rightarrow \infty} \beta_{n}=$ $\beta, x_{n}-v_{n} \rightarrow 0$ and $x_{n}-z_{n} \rightarrow 0$ we deduce that

$$
\lim _{n \rightarrow \infty}\left\|x_{n}-W x_{n}\right\|=0
$$

Noticing $y_{n}=P_{C}\left(u_{n}-\ell_{n} A u_{n}\right)$, we get $\left\langle u_{n}-\ell_{n} A u_{n}-y_{n}, x-y_{n}\right\rangle \leq 0 \forall x \in C$, and hence

$$
\frac{1}{\ell_{n}}\left\langle u_{n}-y_{n}, x-y_{n}\right\rangle+\left\langle A u_{n}, y_{n}-u_{n}\right\rangle \leq\left\langle A u_{n}, x-u_{n}\right\rangle \quad \forall x \in C .
$$

Then, by the boundedness of $\left\{u_{n_{k}}\right\}$ and Lipschitzian property of $A$, we know that $\left\{A u_{n_{k}}\right\}$ is bounded. Also, from $u_{n}-y_{n} \rightarrow 0$, we have that $\left\{y_{n_{k}}\right\}$ is bounded as well. Observe that $\ell_{n} \geq \min \left\{\gamma, \frac{\mu l}{L}\right\}$. So, from (13), it follows that $\liminf _{k \rightarrow \infty}\left\langle A u_{n_{k}}, x-u_{n_{k}}\right\rangle \geq 0 \forall x \in C$. Moreover, note that $\left\langle A y_{n}, x-y_{n}\right\rangle=$ $\left\langle A y_{n}-A u_{n}, x-u_{n}\right\rangle+\left\langle A u_{n}, x-u_{n}\right\rangle+\left\langle A y_{n}, u_{n}-y_{n}\right\rangle$. Since $u_{n}-y_{n} \rightarrow 0$, from $L$-Lipschitzian property of $A$ we get $A u_{n}-A y_{n} \rightarrow 0$, which together with (13) arrives at $\lim _{i n f} \inf _{k \rightarrow \infty}\left\langle A y_{n_{k}}, x-y_{n_{k}}\right\rangle \geq 0 \forall x \in C$. We below claim that $x_{n}-T x_{n} \rightarrow 0$. Indeed, observe that

$$
\begin{aligned}
\left\|T x_{n}-x_{n}\right\| & \leq\left\|T x_{n}-T^{n+1} x_{n}\right\|+\left\|T^{n+1} x_{n}-T^{n} x_{n}\right\|+\left\|T^{n} x_{n}-x_{n}\right\| \\
& \leq\left(1+\theta_{1}\right)\left\|x_{n}-T^{n} x_{n}\right\|+\left\|T^{n+1} x_{n}-T^{n} x_{n}\right\|+\left\|T^{n} x_{n}-x_{n}\right\| \\
& =\left(2+\theta_{1}\right)\left\|x_{n}-T^{n} x_{n}\right\|+\left\|T^{n+1} x_{n}-T^{n} x_{n}\right\| .
\end{aligned}
$$

Therefore, from (11) and the assumption $T^{n} x_{n}-T^{n+1} x_{n} \rightarrow 0$, we get

$$
\lim _{n \rightarrow \infty}\left\|x_{n}-T x_{n}\right\|=0 .
$$

We now select a sequence $\left\{\epsilon_{k}\right\} \subset(0,1)$ s.t. $\epsilon_{k} \downarrow 0$ as $k \rightarrow \infty$. For every $k \geq 1$, we indicate by $m_{k}$ the smallest natural number s.t.

$$
\left\langle A y_{n_{j}}, x-y_{n_{j}}\right\rangle+\epsilon_{k} \geq 0 \quad \forall j \geq m_{k} .
$$

As $\left\{\epsilon_{k}\right\}$ is decreasing, $\left\{m_{k}\right\}$ obviously is increasing. Considering that $\left\{y_{m_{k}}\right\} \subset C$ ensures $A y_{m_{k}} \neq$ $0 \forall k \geq 1$, we put $v_{m_{k}}=\frac{A y_{m_{k}}}{\left\|A y_{m_{k}}\right\|^{2}}$, we have $\left\langle A y_{m_{k}}, v_{m_{k}}\right\rangle=1 \forall k \geq 1$. Therefore, from (15), we have $\left\langle A y_{m_{k}}, x+\epsilon_{k} v_{m_{k}}-y_{m_{k}}\right\rangle \geq 0 \forall k \geq 1$. Also, from the pseudomonotonicity of $A$ we get $\left\langle A\left(x+\epsilon_{k} v_{m_{k}}\right), x+\right.$ $\left.\epsilon_{k} v_{m_{k}}-y_{m_{k}}\right\rangle \geq 0 \forall k \geq 1$. This means that

$$
\left\langle A x, x-y_{m_{k}}\right\rangle \geq\left\langle A x-A\left(x+\epsilon_{k} v_{m_{k}}\right), x+\epsilon_{k} v_{m_{k}}-y_{m_{k}}\right\rangle-\epsilon_{k}\left\langle A x, v_{m_{k}}\right\rangle \quad \forall k \geq 1 .
$$

We show that $\lim _{k \rightarrow \infty} \epsilon_{k} v_{m_{k}}=0$. In fact, from $u_{n_{k}} \rightarrow z$ and $u_{n}-y_{n} \rightarrow 0$, we get $y_{n_{k}} \rightarrow z$. Hence, $\left\{y_{n}\right\} \subset C$ ensures $z \in C$. Also, since $A$ is sequentially weakly continuous, we infer that $A y_{n_{k}} \rightarrow A z$. So, we get $A z \neq 0$ (otherwise, $z$ is a solution). Utilizing the sequentially weak lower semicontinuity of the norm $\|\cdot\|$, we have $0<\|A z\| \leq \liminf _{k \rightarrow \infty}\left\|A y_{n_{k}}\right\|$. Since $\left\{y_{m_{k}}\right\} \subset\left\{y_{n_{k}}\right\}$ and $\epsilon_{k} \downarrow 0$ as $k \rightarrow \infty$, 
we deduce that $0 \leq \lim \sup _{k \rightarrow \infty}\left\|\epsilon_{k} v_{m_{k}}\right\|=\lim \sup _{k \rightarrow \infty} \frac{\epsilon_{k}}{\left\|A y_{m_{k}}\right\|} \leq \frac{\lim \sup _{k \rightarrow \infty} \epsilon_{k}}{\lim \inf _{k \rightarrow \infty}\left\|A y_{n_{k}}\right\|}=0$. Thus we have $\epsilon_{k} \mu_{m_{k}} \rightarrow 0$.

Finally, we claim $z \in \Omega$. In fact, from $u_{n}-x_{n} \rightarrow 0$ and $u_{n_{k}} \rightarrow z$, we have $x_{n_{k}} \rightarrow z$. By (14) we get $x_{n_{k}}-T x_{n_{k}} \rightarrow 0$. Because Lemma 7 ensures the demiclosedness of $I-T$ at zero, we have $z \in \operatorname{Fix}(T)$. Moreover, using $u_{n}-x_{n} \rightarrow 0$ and $u_{n_{k}} \rightarrow z$, we have $x_{n_{k}} \rightarrow z$. Using (12) we get $x_{n_{k}}-W x_{n_{k}} \rightarrow 0$. Using Lemma 7 we deduce that $I-W$ has the demiclosedness at zero. So, we have $(I-W) z=0$, i.e., $z \in \operatorname{Fix}(W)=\cap_{i=1}^{N} \operatorname{Fix}\left(T_{i}\right)$. In addition, taking $k \rightarrow \infty$, we conclude that the right hand side of (16) tends to zero according to the Lipschitzian property of $A$, the boundedness of $\left\{y_{m_{k}}\right\},\left\{v_{m_{k}}\right\}$ and the $\operatorname{limit}_{\lim _{k \rightarrow \infty}} \epsilon_{k} v_{m_{k}}=0$. Consequently, we get $\langle A y, y-z\rangle=\liminf _{k \rightarrow \infty}\left\langle A y, y-y_{m_{k}}\right\rangle \geq 0 \forall y \in C$. By Lemma 3 we have $z \in \operatorname{VI}(C, A)$. So, $z \in \cap_{i=0}^{N} \operatorname{Fix}\left(T_{i}\right) \cap \operatorname{VI}(C, A)=\Omega$.

Remark 2. It is clear that the boundedness assumption of the generated sequences in Lemma 10 can be disposed with when $T$ is the identity.

Theorem 1. Assume that the sequence $\left\{x_{n}\right\}$ constructed by Algorithm 3 satisfies $T^{n} x_{n}-T^{n+1} x_{n} \rightarrow 0$. Then

$$
x_{n} \rightarrow x^{*} \in \Omega \Leftrightarrow\left\{\begin{array}{l}
x_{n}-x_{n+1} \rightarrow 0 \\
x_{n}-T_{N, n} T_{N-1, n} \cdots T_{1, n} x_{n} \rightarrow 0
\end{array}\right.
$$

where $x^{*} \in \Omega$ is only a solution to the HVI: $\left\langle(v f-\rho F) x^{*}, \omega-x^{*}\right\rangle \leq 0 \forall \omega \in \Omega$.

Proof. We first note that $\limsup _{n \rightarrow \infty} \gamma_{n}<1$ and $\liminf _{n \rightarrow \infty} \gamma_{n}>0$. Then, we may suppose that $\left\{\gamma_{n}\right\} \subset[a, b] \subset(0,1)$. We show that $P_{\Omega}(v f+I-\rho F)$ is a contractive map. In fact, using Lemma 6 we get

$$
\begin{aligned}
\| P_{\Omega}(v f+I-\rho F) u- & P_{\Omega}(v f+I-\rho F) v\|\leq v\| f(u)-f(v)\|+\|(I-\rho F) u-(I-\rho F) v \| \\
& \leq v \delta\|u-v\|+(1-\tau)\|u-v\|=[1-(\tau-v \delta)]\|u-v\| \forall u, v \in H .
\end{aligned}
$$

This means that $P_{\Omega}(v f+I-\rho F)$ has only a fixed point $x^{*} \in H$, i.e., $x^{*}=P_{\Omega}(v f+I-\rho F) x^{*}$. Accordingly, there is only a solution $x^{*} \in \Omega=\cap_{i=0}^{N} \operatorname{Fix}\left(T_{i}\right) \cap \operatorname{VI}(C, A)$ to the VIP

$$
\left\langle(v f-\rho F) x^{*}, \omega-x^{*}\right\rangle \leq 0 \quad \forall \omega \in \Omega .
$$

It is now easy to see that the necessity of the theorem is valid. Indeed, if $x_{n} \rightarrow x^{*} \in \Omega=\cap_{i=0}^{N} \operatorname{Fix}\left(T_{i}\right) \cap$ $\operatorname{VI}(C, A)$, then $T_{1} x^{*}=x^{*}, \ldots, T_{N} x^{*}=x^{*}$, which together with $\cap_{i=1}^{N} \operatorname{Fix}\left(T_{i}\right)=\cap_{i=1}^{N} \operatorname{Fix}\left(T_{i, n}\right)=$ $\operatorname{Fix}\left(T_{N}^{n} T_{N-1}^{n} \cdots T_{1}^{n}\right)$ (due to Lemmas 1 and 5), imply that $\left\|x_{n}-x_{n+1}\right\| \leq\left\|x_{n}-x^{*}\right\|+\left\|x_{n+1}-x^{*}\right\| \rightarrow$ $0(n \rightarrow \infty)$, and

$$
\begin{aligned}
\left\|x_{n}-T_{N}^{n} T_{N-1}^{n} \cdots T_{1}^{n} x_{n}\right\| & \leq\left\|x_{n}-x^{*}\right\|+\left\|T_{N}^{n} T_{N-1}^{n} \cdots T_{1}^{n} x_{n}-x^{*}\right\| \\
& \leq\left\|x_{n}-x^{*}\right\|+\left\|x_{n}-x^{*}\right\|=2\left\|x_{n}-x^{*}\right\| \rightarrow 0 \quad(n \rightarrow \infty) .
\end{aligned}
$$

We below claim the sufficiency of the theorem. For this purpose, we suppose $\lim _{n \rightarrow \infty}\left(\left\|x_{n}-x_{n+1}\right\|+\right.$ $\left.\left\|x_{n}-T_{N, n} T_{N-1, n} \cdots T_{1, n} x_{n}\right\|\right)=0$ and prove the sufficiency by the following steps.

Step 1. We claim the boundedness of $\left\{x_{n}\right\}$. In fact, noticing $\lim _{n \rightarrow \infty} \frac{\theta_{n}}{\alpha_{n}}=0$, we know that $\theta_{n} \leq$ $\frac{\alpha_{n}(\tau-v \delta)}{2} \forall n \geq n_{0}$ for some $n_{0} \geq 1$. Therefore, we have that for all $n \geq n_{0}$,

$$
\alpha_{n} v \delta+\gamma_{n}+\left(1-\gamma_{n}-\alpha_{n} \tau\right)\left(1+\theta_{n}\right) \leq 1-\alpha_{n}(\tau-v \delta)+\theta_{n} \leq 1-\frac{\alpha_{n}(\tau-v \delta)}{2} .
$$


Let $p$ be an arbitrary point in $\Omega=\cap \cap_{i=0}^{N} \operatorname{Fix}\left(T_{i}\right) \cap \operatorname{VI}(C, A)$. Then $T p=p, T_{i} p=p, i=1, \ldots, N$, and (10) is true, that is,

$$
\left\|v_{n}-p\right\|^{2}+(1-\mu)\left\|u_{n}-y_{n}\right\|^{2}+(1-\mu)\left\|v_{n}-y_{n}\right\|^{2} \leq\left\|u_{n}-p\right\|^{2} .
$$

Thus, we obtain

$$
\left\|v_{n}-p\right\| \leq\left\|u_{n}-p\right\| \quad \forall n \geq 1
$$

From the definition of $u_{n}$, we have

$$
\left\|u_{n}-p\right\| \leq\left\|x_{n}-p\right\|+\sigma_{n}\left\|x_{n}-x_{n-1}\right\|=\left\|x_{n}-p\right\|+\alpha_{n} \cdot \frac{\sigma_{n}}{\alpha_{n}}\left\|x_{n}-x_{n-1}\right\| .
$$

From $\sup _{n \geq 1} \frac{\sigma_{n}}{\alpha_{n}}<\infty$ and $\sup _{n \geq 1}\left\|x_{n}-x_{n-1}\right\|<\infty$, we infer that $\sup _{n \geq 1} \frac{\sigma_{n}}{\alpha_{n}}\left\|x_{n}-x_{n-1}\right\|<\infty$, which immediately yields that $\exists M_{1}>0$ s.t.

$$
\frac{\sigma_{n}}{\alpha_{n}}\left\|x_{n}-x_{n-1}\right\| \leq M_{1} \quad \forall n \geq 1
$$

Using (19)-(21), we obtain

$$
\left\|v_{n}-p\right\| \leq\left\|u_{n}-p\right\| \leq\left\|x_{n}-p\right\|+\alpha_{n} M_{1} \quad \forall n \geq 1
$$

Accordingly, by Algorithm 3, Lemma 6 and (22) we conclude that for all $n \geq n_{0}$,

$$
\begin{aligned}
& \left\|z_{n}-p\right\|=\left\|\left(1-\beta_{n}\right)\left(T_{N, n} T_{N-1, n} \cdots T_{1, n} v_{n}-p\right)+\beta_{n}\left(x_{n}-p\right)\right\| \\
& \leq\left(1-\beta_{n}\right)\left\|T_{N, n} T_{N-1, n} \cdots T_{1, n} v_{n}-p\right\|+\beta_{n}\left\|x_{n}-p\right\| \\
& \leq\left(1-\beta_{n}\right)\left(\left\|x_{n}-p\right\|+\alpha_{n} M_{1}\right)+\beta_{n}\left\|x_{n}-p\right\| \leq\left\|x_{n}-p\right\|+\alpha_{n} M_{1},
\end{aligned}
$$

and therefore

$$
\begin{aligned}
& \left\|x_{n+1}-p\right\|=\| \gamma_{n}\left(x_{n}-p\right)+\alpha_{n}\left(v f\left(x_{n}\right)-\rho F p\right)+\left(\left(1-\gamma_{n}\right) I-\alpha_{n} \rho F\right) T^{n} z_{n} \\
& -\left(\left(1-\gamma_{n}\right) I-\alpha_{n} \rho F\right) p \| \\
& \leq \alpha_{n} v \delta\left\|x_{n}-p\right\|+\alpha_{n}\|(v f-\rho F) p\|+\gamma_{n}\left\|x_{n}-p\right\| \\
& +\left\|\left(\left(1-\gamma_{n}\right) I-\alpha_{n} \rho F\right) T^{n} z_{n}-\left(\left(1-\gamma_{n}\right) I-\alpha_{n} \rho F\right) p\right\| \\
& =\alpha_{n} v \delta\left\|x_{n}-p\right\|+\alpha_{n}\|(v f-\rho F) p\|+\gamma_{n}\left\|x_{n}-p\right\| \\
& +\left(1-\gamma_{n}\right)\left\|\left(I-\frac{\alpha_{n}}{1-\gamma_{n}} \rho F\right) T^{n} z_{n}-\left(I-\frac{\alpha_{n}}{1-\gamma_{n}} \rho F\right) p\right\| \\
& \leq \alpha_{n} v \delta\left\|x_{n}-p\right\|+\alpha_{n}\|(v f-\rho F) p\|+\gamma_{n}\left\|x_{n}-p\right\| \\
& +\left(1-\gamma_{n}\right)\left(1-\frac{\alpha_{n}}{1-\gamma_{n}} \tau\right)\left(1+\theta_{n}\right)\left\|z_{n}-p\right\| \\
& \leq \alpha_{n} v \delta\left\|x_{n}-p\right\|+\alpha_{n}\|(v f-\rho F) p\| \\
& +\gamma_{n}\left\|x_{n}-p\right\|+\left(1-\gamma_{n}-\alpha_{n} \tau\right)\left(1+\theta_{n}\right)\left(\left\|x_{n}-p\right\|+\alpha_{n} M_{1}\right) \\
& =\left[\alpha_{n} v \delta+\gamma_{n}+\left(1-\gamma_{n}-\alpha_{n} \tau\right)\left(1+\theta_{n}\right)\right]\left\|x_{n}-p\right\| \\
& +\left(1-\gamma_{n}-\alpha_{n} \tau\right)\left(1+\theta_{n}\right) \alpha_{n} M_{1}+\alpha_{n}\|(v f-\rho F) p\| \\
& \leq\left[1-\frac{\alpha_{n}(\tau-v \delta)}{2}\right]\left\|x_{n}-p\right\|+\frac{\alpha_{n}(\tau-v \delta)}{2} \cdot \frac{2\left(M_{1}+\|(v f-\rho F) p\|\right)}{\tau-v \delta} \\
& \leq \max \left\{\frac{2\left(M_{1}+\|(v f-\rho F) p\|\right)}{\tau-v \delta},\left\|x_{n}-p\right\|\right\} \text {. }
\end{aligned}
$$

By induction, we conclude that $\left\|x_{n}-p\right\| \leq \max \left\{\frac{2\left(M_{1}+\|(\rho F-v f) p\|\right)}{\tau-v \delta},\left\|x_{n_{0}}-p\right\|\right\} \forall n \geq n_{0}$. Therefore, we get the boundedness of vector sequence $\left\{x_{n}\right\}$.

Step 2. We claim that $\exists M_{4}>0$ s.t. $\forall n \geq n_{0}$,

$$
\left(1-\gamma_{n}-\alpha_{n} \tau\right)\left(1-\beta_{n}\right)\left(1+\theta_{n}\right)(1-\mu)\left[\left\|u_{n}-y_{n}\right\|^{2}+\left\|v_{n}-y_{n}\right\|^{2}\right] \leq\left\|x_{n}-p\right\|^{2}-\left\|x_{n+1}-p\right\|^{2}+\alpha_{n} M_{4} .
$$


In fact, using Lemma 6, Lemma 9, and the convexity of $\|\cdot\|^{2}$, from $\alpha_{n}+\gamma_{n} \leq 1$, we obtain that for all $n \geq n_{0}$,

$$
\begin{aligned}
&\left\|x_{n+1}-p\right\|^{2}=\| \alpha_{n} v\left(f\left(x_{n}\right)-f(p)\right)+\gamma_{n}\left(x_{n}-p\right)+\left(\left(1-\gamma_{n}\right) I-\alpha_{n} \rho F\right) T^{n} z_{n} \\
& \quad-\left(\left(1-\gamma_{n}\right) I-\alpha_{n} \rho F\right) p+\alpha_{n}(v f-\rho F) p \|^{2} \\
& \leq \| \alpha_{n} v\left(f\left(x_{n}\right)-f(p)\right)+\gamma_{n}\left(x_{n}-p\right)+\left(\left(1-\gamma_{n}\right) I-\alpha_{n} \rho F\right) T^{n} z_{n} \\
&-\left(\left(1-\gamma_{n}\right) I-\alpha_{n} \rho F\right) p \|^{2}+2 \alpha_{n}\left\langle(v f-\rho F) p, x_{n+1}-p\right\rangle \\
&= \| \alpha_{n} v\left(f\left(x_{n}\right)-f(p)\right)+\gamma_{n}\left(x_{n}-p\right)+\left(1-\gamma_{n}\right)\left[\left(I-\frac{\alpha_{n}}{1-\gamma_{n}} \rho F\right) T^{n} z_{n}\right. \\
&\left.-\left(I-\frac{\alpha_{n}}{1-\gamma_{n}} \rho F\right) p\right] \|^{2}+2 \alpha_{n}\left\langle(v f-\rho F) p, x_{n+1}-p\right\rangle \\
& \leq {\left[\alpha_{n} v \delta\left\|x_{n}-p\right\|+\gamma_{n}\left\|x_{n}-p\right\|+\left(1-\gamma_{n}\right)\left(1-\frac{\alpha_{n}}{1-\gamma_{n}} \tau\right)\left(1+\theta_{n}\right)\left\|z_{n}-p\right\|\right]^{2} } \\
&+2 \alpha_{n}\left\langle(v f-\rho F) p, x_{n+1}-p\right\rangle \\
& \leq \alpha_{n} v \delta\left\|x_{n}-p\right\|^{2}+\gamma_{n}\left\|x_{n}-p\right\|^{2}+\left(1-\gamma_{n}-\alpha_{n} \tau\right)\left(1+\theta_{n}\right)\left\|z_{n}-p\right\|^{2} \\
&+2 \alpha_{n}\left\langle(v f-\rho F) p, x_{n+1}-p\right\rangle \\
& \leq \alpha_{n} v \delta\left\|x_{n}-p\right\|^{2}+\gamma_{n}\left\|x_{n}-p\right\|^{2}+\left(1-\gamma_{n}-\alpha_{n} \tau\right)\left(1+\theta_{n}\right)\left[\beta_{n}\left\|x_{n}-p\right\|^{2}\right. \\
&\left.+\left(1-\beta_{n}\right)\left\|u_{n}-p\right\|^{2}-\left(1-\beta_{n}\right)(1-\mu)\left(\left\|u_{n}-y_{n}\right\|^{2}+\left\|v_{n}-y_{n}\right\|^{2}\right)\right]+\alpha_{n} M_{2},
\end{aligned}
$$

where $\sup _{n \geq 1} 2\|(v f-\rho F) p\|\left\|x_{n+1}-p\right\| \leq M_{2}$ for some $M_{2}>0$. Also, from (22), we get

$$
\left\|u_{n}-p\right\|^{2} \leq\left\|x_{n}-p\right\|^{2}+\alpha_{n}\left(2 M_{1}\left\|x_{n}-p\right\|+\alpha_{n} M_{1}^{2}\right) \leq\left\|x_{n}-p\right\|^{2}+\alpha_{n} M_{3},
$$

where $\sup _{n \geq 1}\left\{2 M_{1}\left\|x_{n}-p\right\|+\alpha_{n} M_{1}^{2}\right\} \leq M_{3}$ for some $M_{3}>0$. Note that $\alpha_{n} v \delta+\gamma_{n}+\left(1-\gamma_{n}-\right.$ $\left.\alpha_{n} \tau\right)\left(1+\theta_{n}\right) \leq 1-\frac{\alpha_{n}(\tau-v \delta)}{2}$ for all $n \geq n_{0}$. Substituting (25) for (24), we deduce that for all $n \geq n_{0}$,

$$
\begin{aligned}
& \left\|x_{n+1}-p\right\|^{2} \\
\leq & \alpha_{n} v \delta\left\|x_{n}-p\right\|^{2}+\gamma_{n}\left\|x_{n}-p\right\|^{2}+\left(1-\gamma_{n}-\alpha_{n} \tau\right)\left(1+\theta_{n}\right)\left[\beta_{n}\left\|x_{n}-p\right\|^{2}\right. \\
& \left.+\left(1-\beta_{n}\right)\left(\left\|x_{n}-p\right\|^{2}+\alpha_{n} M_{3}\right)-\left(1-\beta_{n}\right)(1-\mu)\left(\left\|u_{n}-y_{n}\right\|^{2}+\left\|v_{n}-y_{n}\right\|^{2}\right)\right]+\alpha_{n} M_{2} \\
\leq & {\left[\alpha_{n} v \delta+\gamma_{n}+\left(1-\gamma_{n}-\alpha_{n} \tau\right)\left(1+\theta_{n}\right)\right]\left\|x_{n}-p\right\|^{2} } \\
& +\left(1-\gamma_{n}-\alpha_{n} \tau\right)\left(1+\theta_{n}\right)\left[\alpha_{n} M_{3}-\left(1-\beta_{n}\right)(1-\mu)\left(\left\|u_{n}-y_{n}\right\|^{2}+\left\|v_{n}-y_{n}\right\|^{2}\right)\right]+\alpha_{n} M_{2} \\
\leq & \left(1-\frac{\alpha_{n}(\tau-v \delta)}{2}\right)\left\|x_{n}-p\right\|^{2}-\left(1-\gamma_{n}-\alpha_{n} \tau\right)\left(1-\beta_{n}\right)\left(1+\theta_{n}\right)(1-\mu) \times \\
& \times\left[\left\|u_{n}-y_{n}\right\|^{2}+\left\|v_{n}-y_{n}\right\|^{2}\right]+\alpha_{n} M_{2}+\alpha_{n} M_{3} \\
\leq & \left\|x_{n}-p\right\|^{2}-\left(1-\gamma_{n}-\alpha_{n} \tau\right)\left(1-\beta_{n}\right)\left(1+\theta_{n}\right)(1-\mu)\left[\left\|u_{n}-y_{n}\right\|^{2}+\left\|v_{n}-y_{n}\right\|^{2}\right]+\alpha_{n} M_{4},
\end{aligned}
$$

where $M_{4}:=M_{2}+M_{3}$. This immediately implies that for all $n \geq n_{0}$,

$$
\left(1-\gamma_{n}-\alpha_{n} \tau\right)\left(1-\beta_{n}\right)\left(1+\theta_{n}\right)(1-\mu)\left[\left\|u_{n}-y_{n}\right\|^{2}+\left\|v_{n}-y_{n}\right\|^{2}\right] \leq\left\|x_{n}-p\right\|^{2}-\left\|x_{n+1}-p\right\|^{2}+\alpha_{n} M_{4} .
$$

Step 3. We claim that $\exists M>0$ s.t. $\forall n \geq n_{0}$,

$$
\begin{aligned}
\left\|x_{n+1}-p\right\|^{2} \leq & {\left[1-\frac{\alpha_{n}(\tau-v \delta)}{2}\right]\left\|x_{n}-p\right\|^{2}+\frac{\alpha_{n}(\tau-v \delta)}{2}\left[\frac{4}{\tau-v \delta}\left\langle(v f-\rho F) p, x_{n+1}-p\right\rangle\right.} \\
& \left.+\frac{\sigma_{n}}{\alpha_{n}} \cdot \frac{2 M}{\tau-v \delta}\left\|x_{n}-x_{n-1}\right\|\right] .
\end{aligned}
$$

In fact, we get

$$
\left\|u_{n}-p\right\|^{2} \leq\left(\left\|x_{n}-p\right\|+\sigma_{n}\left\|x_{n}-x_{n-1}\right\|\right)^{2} \leq\left\|x_{n}-p\right\|^{2}+\sigma_{n}\left\|x_{n}-x_{n-1}\right\| M,
$$

with $\sup _{n \geq 1}\left\{2\left\|x_{n}-p\right\|+\sigma_{n}\left\|x_{n}-x_{n-1}\right\|\right\} \leq M$ for some $M>0$. Note that $\alpha_{n} v \delta+\gamma_{n}+\left(1-\gamma_{n}-\right.$ $\left.\alpha_{n} \tau\right)\left(1+\theta_{n}\right) \leq 1-\frac{\alpha_{n}(\tau-v \delta)}{2}$ for all $n \geq n_{0}$. Thus, combining (24) and (27), we have that for all $n \geq n_{0}$, 


$$
\begin{aligned}
\left\|x_{n+1}-p\right\|^{2} \leq & \alpha_{n} v \delta\left\|x_{n}-p\right\|^{2}+\gamma_{n}\left\|x_{n}-p\right\|^{2}+\left(1-\gamma_{n}-\alpha_{n} \tau\right)\left(1+\theta_{n}\right)\left[\left\|x_{n}-p\right\|^{2}\right. \\
& \left.+\sigma_{n}\left\|x_{n}-x_{n-1}\right\| M\right]+2 \alpha_{n}\left\langle(v f-\rho F) p, x_{n+1}-p\right\rangle \\
= & {\left[\alpha_{n} v \delta+\gamma_{n}+\left(1-\gamma_{n}-\alpha_{n} \tau\right)\left(1+\theta_{n}\right)\right]\left\|x_{n}-p\right\|^{2} } \\
& +\left(1-\gamma_{n}-\alpha_{n} \tau\right)\left(1+\theta_{n}\right) \sigma_{n}\left\|x_{n}-x_{n-1}\right\| M+2 \alpha_{n}\left\langle(v f-\rho F) p, x_{n+1}-p\right\rangle \\
\leq & {\left[1-\frac{\alpha_{n}(\tau-v \delta)}{2}\right]\left\|x_{n}-p\right\|^{2}+\frac{\alpha_{n}(\tau-v \delta)}{2}\left[\frac{\left.4(v f-\rho F) p, x_{n+1}-p\right\rangle}{\tau-v \delta}+\frac{\sigma_{n}}{\alpha_{n}} \cdot \frac{\left\|x_{n}-x_{n-1}\right\| 2 M}{\tau-v \delta}\right] . }
\end{aligned}
$$

Step 4. We claim that $x_{n} \rightarrow x^{*} \in \Omega$, which is only a solution to the VIP (17). In fact, setting $p=x^{*}$, we obtain from (28) that

$$
\begin{aligned}
\left\|x_{n+1}-x^{*}\right\|^{2} \leq & {\left[1-\frac{\alpha_{n}(\tau-v \delta)}{2}\right]\left\|x_{n}-x^{*}\right\|^{2}+\frac{\alpha_{n}(\tau-v \delta)}{2}\left[\frac{4}{\tau-v \delta}\left\langle(v f-\rho F) x^{*}, x_{n+1}-x^{*}\right\rangle\right.} \\
& \left.+\frac{\sigma_{n}}{\alpha_{n}} \cdot \frac{2 M}{\tau-v \delta}\left\|x_{n}-x_{n-1}\right\|\right] .
\end{aligned}
$$

According to Lemma 4 , it is sufficient to prove that $\limsup _{n \rightarrow \infty}\left\langle(v f-\rho F) x^{*}, x_{n+1}-x^{*}\right\rangle \leq 0$. As $x_{n}-x_{n+1} \rightarrow 0, \alpha_{n} \rightarrow 0, \beta_{n} \rightarrow \beta<1$ and $\theta_{n} \rightarrow 0$, from (26) and $\left\{\gamma_{n}\right\} \subset[a, b] \subset(0,1)$, we have

$$
\begin{aligned}
& \limsup _{n \rightarrow \infty}\left(1-b-\alpha_{n} \tau\right)\left(1-\beta_{n}\right)\left(1+\theta_{n}\right)(1-\mu)\left[\left\|u_{n}-y_{n}\right\|^{2}+\left\|v_{n}-y_{n}\right\|^{2}\right] \\
& \leq \limsup _{n \rightarrow \infty}\left(1-\gamma_{n}-\alpha_{n} \tau\right)\left(1-\beta_{n}\right)\left(1+\theta_{n}\right)(1-\mu)\left[\left\|u_{n}-y_{n}\right\|^{2}+\left\|v_{n}-y_{n}\right\|^{2}\right] \\
& \leq \limsup _{n \rightarrow \infty}\left(\left\|x_{n}-p\right\|+\left\|x_{n+1}-p\right\|\right)\left\|x_{n}-x_{n+1}\right\|=0 .
\end{aligned}
$$

This immediately implies that

$$
\lim _{n \rightarrow \infty}\left\|u_{n}-y_{n}\right\|=0 \quad \text { and } \quad \lim _{n \rightarrow \infty}\left\|v_{n}-y_{n}\right\|=0 .
$$

In addition, it is clear that $\left\|u_{n}-x_{n}\right\|=\sigma_{n}\left\|x_{n}-x_{n-1}\right\| \leq\left\|x_{n}-x_{n-1}\right\| \rightarrow 0(n \rightarrow \infty)$, and hence $\left\|x_{n}-y_{n}\right\| \leq\left\|x_{n}-u_{n}\right\|+\left\|u_{n}-y_{n}\right\| \rightarrow 0(n \rightarrow \infty)$. So it follows from (30) that $\left\|x_{n}-v_{n}\right\| \leq\left\|x_{n}-y_{n}\right\|+$ $\left\|y_{n}-v_{n}\right\| \rightarrow 0(n \rightarrow \infty)$. Thus, from Algorithm 3 and the assumption $x_{n}-T_{N}^{n} T_{N-1}^{n} \cdots T_{1}^{n} x_{n} \rightarrow 0$, we obtain

$$
\begin{aligned}
& \left\|z_{n}-x_{n}\right\|=\left(1-\beta_{n}\right)\left\|T_{N, n} T_{N-1, n} \cdots T_{1, n} v_{n}-x_{n}\right\| \leq\left\|T_{N, n} T_{N-1, n} \cdots T_{1, n} v_{n}-x_{n}\right\| \\
& \leq\left\|T_{N, n} T_{N-1, n} \cdots T_{1, n} v_{n}-T_{N, n} T_{N-1, n} \cdots T_{1, n} x_{n}\right\|+\left\|T_{N, n} T_{N-1, n} \cdots T_{1, n} x_{n}-x_{n}\right\| \\
& \leq\left\|v_{n}-x_{n}\right\|+\left\|T_{N, n} T_{N-1, n} \cdots T_{1, n} x_{n}-x_{n}\right\| \rightarrow 0 \quad(n \rightarrow \infty)
\end{aligned}
$$

As $x_{n}-y_{n} \rightarrow 0, x_{n}-z_{n} \rightarrow 0$ and $u_{n}-x_{n} \rightarrow 0$, we deduce that as $n \rightarrow \infty$,

$$
\left\|u_{n}-y_{n}\right\| \leq\left\|u_{n}-x_{n}\right\|+\left\|x_{n}-y_{n}\right\| \rightarrow 0 \quad \text { and } \quad\left\|u_{n}-z_{n}\right\| \leq\left\|u_{n}-x_{n}\right\|+\left\|x_{n}-z_{n}\right\| \rightarrow 0 .
$$

On the other hand, from the boundedness of $\left\{x_{n}\right\}$, it follows that $\exists\left\{x_{n_{k}}\right\} \subset\left\{x_{n}\right\}$ s.t.

$$
\limsup _{n \rightarrow \infty}\left\langle(v f-\rho F) x^{*}, x_{n}-x^{*}\right\rangle=\lim _{k \rightarrow \infty}\left\langle(v f-\rho F) x^{*}, x_{n_{k}}-x^{*}\right\rangle .
$$

Utilizing the reflexivity of $H$ and the boundedness of $\left\{x_{n}\right\}$, one may suppose that $x_{n_{k}} \rightarrow \tilde{x}$. Therefore, one gets from (33),

$$
\limsup _{n \rightarrow \infty}\left\langle(v f-\rho F) x^{*}, x_{n}-x^{*}\right\rangle=\left\langle(v f-\rho F) x^{*}, \tilde{x}-x^{*}\right\rangle .
$$

It is easy to see from $u_{n}-x_{n} \rightarrow 0$ and $x_{n_{k}} \rightarrow \tilde{x}$ that $w_{n_{k}} \rightarrow \tilde{x}$. Since $T^{n} x_{n}-T^{n+1} x_{n} \rightarrow 0, x_{n}-x_{n+1} \rightarrow$ $0, u_{n}-y_{n} \rightarrow 0, u_{n}-z_{n} \rightarrow 0$ and $w_{n_{k}} \rightarrow \tilde{x}$, from Lemma 10 we get $\tilde{x} \in \Omega$. Therefore, from (17) and (34), we infer that

$$
\limsup _{n \rightarrow \infty}\left\langle(v f-\rho F) x^{*}, x_{n}-x^{*}\right\rangle=\left\langle(v f-\rho F) x^{*}, \tilde{x}-x^{*}\right\rangle \leq 0,
$$


which together with $x_{n}-x_{n+1} \rightarrow 0$, implies that

$$
\begin{aligned}
& \limsup _{n \rightarrow \infty}\left\langle(v f-\rho F) x^{*}, x_{n+1}-x^{*}\right\rangle \\
& =\limsup _{n \rightarrow \infty}\left[\left\langle(v f-\rho F) x^{*}, x_{n+1}-x_{n}\right\rangle+\left\langle(v f-\rho F) x^{*}, x_{n}-x^{*}\right\rangle\right] \\
& =\left\langle(v f-\rho F) x^{*}, \tilde{x}-x^{*}\right\rangle \leq 0 .
\end{aligned}
$$

Observe that $\left\{\frac{\alpha_{n}(\tau-v \delta)}{2}\right\} \subset[0,1], \sum_{n=1}^{\infty} \frac{\alpha_{n}(\tau-v \delta)}{2}=\infty$, and

$$
\limsup _{n \rightarrow \infty}\left[\frac{4}{\tau-v \delta}\left\langle(v f-\rho F) x^{*}, x_{n+1}-x^{*}\right\rangle+\frac{\sigma_{n}}{\alpha_{n}} \cdot \frac{2 M}{\tau-v \delta}\left\|x_{n}-x_{n-1}\right\|\right] \leq 0 .
$$

Consequently, by Lemma 4 we obtain from (29) that $\left\|x_{n}-x^{*}\right\| \rightarrow 0$ as $n \rightarrow \infty$.

Next, we introduce another mildly inertial subgradient extragradient algorithm with line-search process.

It is remarkable that Lemmas 8 and 9 remain true for Algorithm 4.

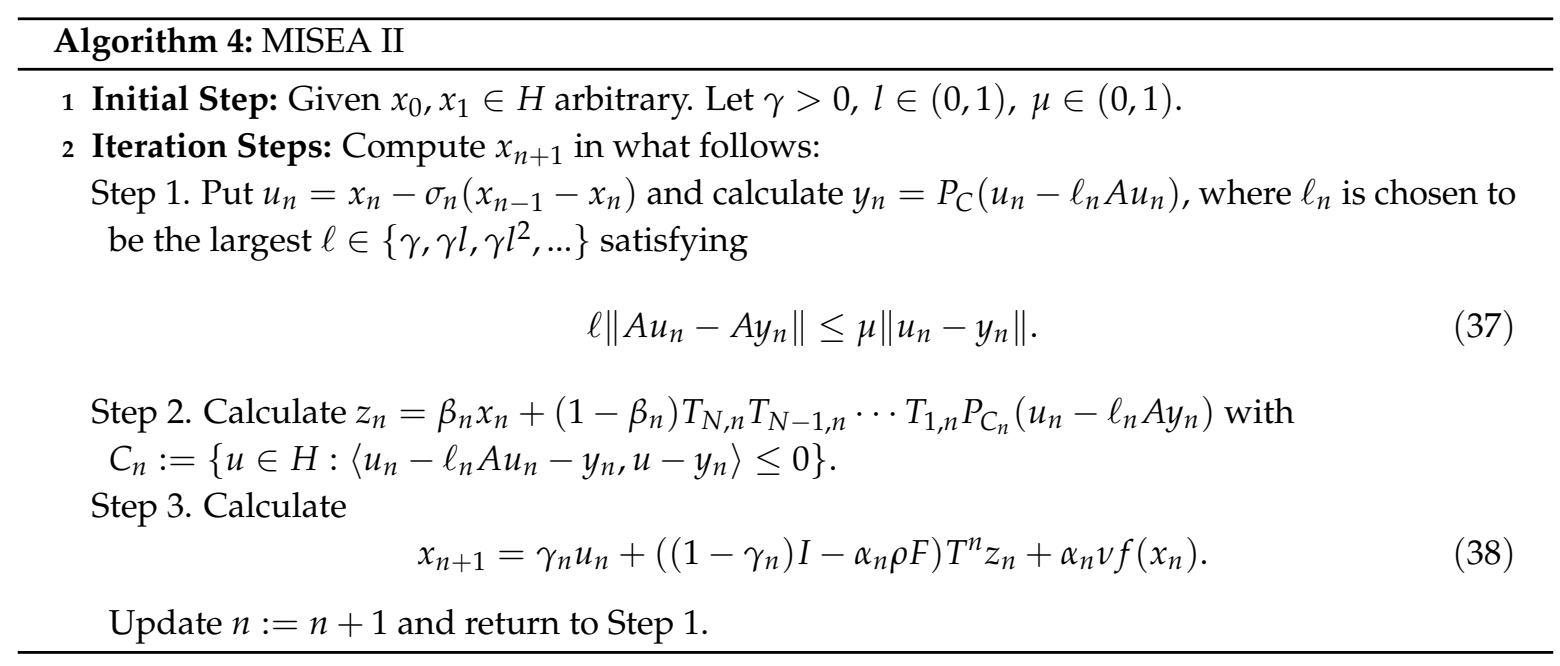

Theorem 2. Assume that the sequence $\left\{x_{n}\right\}$ constructed by Algorithm 4 satisfies $T^{n} x_{n}-T^{n+1} x_{n} \rightarrow 0$. Then,

$$
x_{n} \rightarrow x^{*} \in \Omega \Leftrightarrow\left\{\begin{array}{l}
x_{n}-x_{n+1} \rightarrow 0, \\
x_{n}-T_{N, n} T_{N-1, n} \cdots T_{1, n} x_{n} \rightarrow 0
\end{array}\right.
$$

where $x^{*} \in \Omega$ is only a solution to the HVI: $\left\langle(v f-\rho F) x^{*}, \omega-x^{*}\right\rangle \leq 0 \forall \omega \in \Omega$.

Proof. Using the similar inference to that in the proof of Theorem 1, we obtain that there is only a solution $x^{*} \in \Omega=\cap \cap_{i=0}^{N} \operatorname{Fix}\left(T_{i}\right) \cap \operatorname{VI}(C, A)$ to the HVI (17), and that the necessity of the theorem is true.

We claim the sufficiency of the theorem below. For this purpose, we suppose $\lim _{n \rightarrow \infty}\left(\| x_{n}-\right.$ $\left.x_{n+1}\|+\| x_{n}-T_{N, n} T_{N-1, n} \cdots T_{1, n} x_{n} \|\right)=0$ and prove the sufficiency by the following steps.

Step 1. We claim the boundedness of $\left\{x_{n}\right\}$. In fact, using the similar reasoning to that in Step 1 for the proof of Theorem 1, we know that inequalities (18)-(23) hold. Taking into account $\lim _{n \rightarrow \infty} \frac{\theta_{n}}{\alpha_{n}}=0$, we know that $\theta_{n} \leq \frac{\alpha_{n}(\tau-v \delta)}{2} \forall n \geq n_{0}$ for some $n_{0} \geq 1$. Hence we deduce that for all $n \geq n_{0}$,

$$
\alpha_{n} v \delta+\gamma_{n}+\left(1-\gamma_{n}-\alpha_{n} \tau\right)\left(1+\theta_{n}\right) \leq 1-\alpha_{n}(\tau-v \delta)+\theta_{n} \leq 1-\frac{\alpha_{n}(\tau-v \delta)}{2} .
$$


Also, from Algorithm 4, Lemma 6, and (22) and (23) we obtain

$$
\begin{aligned}
\left\|x_{n+1}-p\right\|= & \| \gamma_{n}\left(u_{n}-p\right)+\alpha_{n}\left(v f\left(x_{n}\right)-\rho F p\right)+\left(\left(1-\gamma_{n}\right) I-\alpha_{n} \rho F\right) T^{n} z_{n} \\
& -\left(\left(1-\gamma_{n}\right) I-\alpha_{n} \rho F\right) p \| \\
\leq & \alpha_{n} v \delta\left\|x_{n}-p\right\|+\alpha_{n}\|(v f-\rho F) p\|+\gamma_{n}\left\|u_{n}-p\right\| \\
& +\left(1-\gamma_{n}\right)\left\|\left(I-\frac{\alpha_{n}}{1-\gamma_{n}} \rho F\right) T^{n} z_{n}-\left(I-\frac{\alpha_{n}}{1-\gamma_{n}} \rho F\right) p\right\| \\
\leq & \alpha_{n} v \delta\left\|x_{n}-p\right\|+\alpha_{n}\|(v f-\rho F) p\|+\gamma_{n}\left\|u_{n}-p\right\| \\
& +\left(1-\gamma_{n}\right)\left(1-\frac{\alpha_{n}}{1-\gamma_{n}} \tau\right)\left(1+\theta_{n}\right)\left\|z_{n}-p\right\| \\
\leq & \alpha_{n} v \delta\left\|x_{n}-p\right\|+\alpha_{n}\|(v f-\rho F) p\| \\
& +\gamma_{n}\left(\left\|x_{n}-p\right\|+\alpha_{n} M_{1}\right)+\left(1-\gamma_{n}-\alpha_{n} \tau\right)\left(1+\theta_{n}\right)\left(\left\|x_{n}-p\right\|+\alpha_{n} M_{1}\right) \\
= & {\left[\alpha_{n} v \delta+\gamma_{n}+\left(1-\gamma_{n}-\alpha_{n} \tau\right)\left(1+\theta_{n}\right)\right]\left\|x_{n}-p\right\| } \\
& +\left[\gamma_{n}+\left(1-\gamma_{n}-\alpha_{n} \tau\right)\left(1+\theta_{n}\right)\right] \alpha_{n} M_{1}+\alpha_{n}\|(v f-\rho F) p\| \\
\leq & {\left[1-\frac{\alpha_{n}(\tau-v \delta)}{2}\right]\left\|x_{n}-p\right\|+\frac{\alpha_{n}(\tau-v \delta)}{2} \cdot \frac{2\left(M_{1}+\|(v f-\rho F) p\|\right)}{\tau-v \delta} } \\
\leq & \max \left\{\frac{2\left(M_{1}+\|(v f-\rho F) p\|\right)}{\tau-v \delta},\left\|x_{n}-p\right\|\right\} .
\end{aligned}
$$

By induction, we conclude that $\left\|x_{n}-p\right\| \leq \max \left\{\frac{2\left(M_{1}+\|(\rho F-v f) p\|\right)}{\tau-v \delta},\left\|x_{n_{0}}-p\right\|\right\} \forall n \geq n_{0}$. Therefore, we obtain the boundedness of vector sequence $\left\{x_{n}\right\}$.

Step 2. One claims $\exists M_{4}>0$ s.t. $\forall n \geq n_{0}$,

$$
\left(1-\gamma_{n}-\alpha_{n} \tau\right)\left(1-\beta_{n}\right)\left(1+\theta_{n}\right)(1-\mu)\left[\left\|u_{n}-y_{n}\right\|^{2}+\left\|v_{n}-y_{n}\right\|^{2}\right] \leq\left\|x_{n}-p\right\|^{2}-\left\|x_{n+1}-p\right\|^{2}+\alpha_{n} M_{4} .
$$

In fact, using Lemma 6, Lemma 9, and the convexity of $\|\cdot\|^{2}$, from $\alpha_{n}+\gamma_{n} \leq 1$, we obtain that for all $n \geq n_{0}$,

$$
\begin{aligned}
\| & x_{n+1}-p\left\|^{2}=\right\| \gamma_{n}\left(u_{n}-p\right)+\alpha_{n} v\left(f\left(x_{n}\right)-f(p)\right)+\left(\left(1-\gamma_{n}\right) I-\alpha_{n} \rho F\right) T^{n} z_{n} \\
& -\left(\left(1-\gamma_{n}\right) I-\alpha_{n} \rho F\right) p+\alpha_{n}(v f-\rho F) p \|^{2} \\
\leq & \| \alpha_{n} v\left(f\left(x_{n}\right)-f(p)\right)+\gamma_{n}\left(u_{n}-p\right)+\left(\left(1-\gamma_{n}\right) I-\alpha_{n} \rho F\right) T^{n} z_{n} \\
& -\left(\left(1-\gamma_{n}\right) I-\alpha_{n} \rho F\right) p \|^{2}+2 \alpha_{n}\left\langle(v f-\rho F) p, x_{n+1}-p\right\rangle \\
\leq & {\left[\alpha_{n} v \delta\left\|x_{n}-p\right\|+\gamma_{n}\left\|u_{n}-p\right\|+\left(1-\gamma_{n}-\alpha_{n} \tau\right)\left(1+\theta_{n}\right)\left\|z_{n}-p\right\|\right]^{2} } \\
& +2 \alpha_{n}\left\langle(v f-\rho F) p, x_{n+1}-p\right\rangle \\
\leq & \alpha_{n} v \delta\left\|x_{n}-p\right\|^{2}+\gamma_{n}\left\|u_{n}-p\right\|^{2}+\left(1-\gamma_{n}-\alpha_{n} \tau\right)\left(1+\theta_{n}\right)\left\|z_{n}-p\right\|^{2} \\
& +2 \alpha_{n}\left\langle(v f-\rho F) p, x_{n+1}-p\right\rangle \\
\leq & \alpha_{n} v \delta\left\|x_{n}-p\right\|^{2}+\gamma_{n}\left\|u_{n}-p\right\|^{2}+\left(1-\gamma_{n}-\alpha_{n} \tau\right)\left(1+\theta_{n}\right)\left[\beta_{n}\left\|x_{n}-p\right\|^{2}\right. \\
& \left.+\left(1-\beta_{n}\right)\left\|u_{n}-p\right\|^{2}-\left(1-\beta_{n}\right)(1-\mu)\left(\left\|u_{n}-y_{n}\right\|^{2}+\left\|v_{n}-y_{n}\right\|^{2}\right)\right]+\alpha_{n} M_{2},
\end{aligned}
$$

where $\sup _{n \geq 1} 2\|(v f-\rho F) p\|\left\|x_{n+1}-p\right\| \leq M_{2}$ for some $M_{2}>0$. Also, from (22) we have

$$
\left\|u_{n}-p\right\|^{2} \leq\left\|x_{n}-p\right\|^{2}+\alpha_{n} M_{3}
$$

where $\sup _{n \geq 1}\left\{2 M_{1}\left\|x_{n}-p\right\|+\alpha_{n} M_{1}^{2}\right\} \leq M_{3}$ for some $M_{3}>0$. Note that $\alpha_{n} v \delta+\gamma_{n}+\left(1-\gamma_{n}-\right.$ $\left.\alpha_{n} \tau\right)\left(1+\theta_{n}\right) \leq 1-\frac{\alpha_{n}(\tau-v \delta)}{2}$ for all $n \geq n_{0}$. Substituting (40) for (39), we deduce that for all $n \geq n_{0}$,

$$
\begin{aligned}
& \left\|x_{n+1}-p\right\|^{2} \\
\leq & \gamma_{n}\left(\left\|x_{n}-p\right\|^{2}+\alpha_{n} M_{3}\right)+\alpha_{n} v \delta\left\|x_{n}-p\right\|^{2}+\left(1-\gamma_{n}-\alpha_{n} \tau\right)\left(1+\theta_{n}\right)\left[\left\|x_{n}-p\right\|^{2}\right. \\
& \left.+\alpha_{n} M_{3}-\left(1-\beta_{n}\right)(1-\mu)\left(\left\|u_{n}-y_{n}\right\|^{2}+\left\|v_{n}-y_{n}\right\|^{2}\right)\right]+\alpha_{n} M_{2} \\
= & {\left[\alpha_{n} v \delta+\gamma_{n}+\left(1-\gamma_{n}-\alpha_{n} \tau\right)\left(1+\theta_{n}\right)\right]\left\|x_{n}-p\right\|^{2}+\gamma_{n} \alpha_{n} M_{3} } \\
& +\left(1-\gamma_{n}-\alpha_{n} \tau\right)\left(1+\theta_{n}\right)\left[\alpha_{n} M_{3}-\left(1-\beta_{n}\right)(1-\mu)\left(\left\|u_{n}-y_{n}\right\|^{2}+\left\|v_{n}-y_{n}\right\|^{2}\right)\right]+\alpha_{n} M_{2} \\
\leq & \left(1-\frac{\alpha_{n}(\tau-v \delta)}{2}\right)\left\|x_{n}-p\right\|^{2}-\left(1-\gamma_{n}-\alpha_{n} \tau\right)\left(1-\beta_{n}\right)\left(1+\theta_{n}\right)(1-\mu) \times \\
& \times\left[\left\|u_{n}-y_{n}\right\|^{2}+\left\|v_{n}-y_{n}\right\|^{2}\right]+\alpha_{n} M_{2}+\alpha_{n} M_{3} \\
\leq & \left\|x_{n}-p\right\|^{2}-\left(1-\gamma_{n}-\alpha_{n} \tau\right)\left(1-\beta_{n}\right)\left(1+\theta_{n}\right)(1-\mu)\left[\left\|u_{n}-y_{n}\right\|^{2}+\left\|v_{n}-y_{n}\right\|^{2}\right]+\alpha_{n} M_{4},
\end{aligned}
$$


where $M_{4}:=M_{2}+M_{3}$. This immediately implies that for all $n \geq n_{0}$,

$$
\left(1-\gamma_{n}-\alpha_{n} \tau\right)\left(1-\beta_{n}\right)\left(1+\theta_{n}\right)(1-\mu)\left[\left\|u_{n}-y_{n}\right\|^{2}+\left\|v_{n}-y_{n}\right\|^{2}\right] \leq\left\|x_{n}-p\right\|^{2}-\left\|x_{n+1}-p\right\|^{2}+\alpha_{n} M_{4} .
$$

Step 3. One claims that $\exists M>0$ s.t. $\forall n \geq n_{0}$,

$$
\begin{aligned}
\left\|x_{n+1}-p\right\|^{2} \leq & {\left[1-\frac{\alpha_{n}(\tau-v \delta)}{2}\right]\left\|x_{n}-p\right\|^{2}+\frac{\alpha_{n}(\tau-v \delta)}{2}\left[\frac{4}{\tau-\nu \delta}\left\langle(v f-\rho F) p, x_{n+1}-p\right\rangle\right.} \\
& \left.+\frac{\sigma_{n}}{\alpha_{n}} \cdot \frac{2 M}{\tau-\nu \delta}\left\|x_{n}-x_{n-1}\right\|\right] .
\end{aligned}
$$

In fact, we get

$$
\left\|u_{n}-p\right\|^{2} \leq\left\|x_{n}-p\right\|^{2}+\sigma_{n}\left\|x_{n}-x_{n-1}\right\| M,
$$

where $\sup _{n \geq 1}\left\{2\left\|x_{n}-p\right\|+\sigma_{n}\left\|x_{n}-x_{n-1}\right\|\right\} \leq M$ for some $M>0$. Observe that $\alpha_{n} v \delta+\gamma_{n}+(1-$ $\left.\gamma_{n}-\alpha_{n} \tau\right)\left(1+\theta_{n}\right) \leq 1-\frac{\alpha_{n}(\tau-v \delta)}{2}$ for all $n \geq n_{0}$. Thus, combining (39) and (42), we have that for all $n \geq n_{0}$

$$
\begin{aligned}
& \left\|x_{n+1}-p\right\|^{2} \\
\leq & \gamma_{n}\left(\left\|x_{n}-p\right\|^{2}+\sigma_{n}\left\|x_{n}-x_{n-1}\right\| M\right)+\alpha_{n} v \delta\left\|x_{n}-p\right\|^{2}+\left(1-\gamma_{n}-\alpha_{n} \tau\right)\left(1+\theta_{n}\right)\left[\beta_{n}\left\|x_{n}-p\right\|^{2}\right. \\
& \left.+\left(1-\beta_{n}\right)\left(\left\|x_{n}-p\right\|^{2}+\sigma_{n}\left\|x_{n}-x_{n-1}\right\| M\right)\right]+2 \alpha_{n}\left\langle(v f-\rho F) p, x_{n+1}-p\right\rangle \\
\leq & \gamma_{n}\left(\left\|x_{n}-p\right\|^{2}+\sigma_{n}\left\|x_{n}-x_{n-1}\right\| M\right)+\alpha_{n} v \delta\left\|x_{n}-p\right\|^{2}+\left(1-\gamma_{n}-\alpha_{n} \tau\right)\left(1+\theta_{n}\right)\left[\left\|x_{n}-p\right\|^{2}\right. \\
& \left.+\sigma_{n}\left\|x_{n}-x_{n-1}\right\| M\right]+2 \alpha_{n}\left\langle(v f-\rho F) p, x_{n+1}-p\right\rangle \\
= & {\left[\alpha_{n} v \delta+\gamma_{n}+\left(1-\gamma_{n}-\alpha_{n} \tau\right)\left(1+\theta_{n}\right)\right]\left\|x_{n}-p\right\|^{2}+\gamma_{n} \sigma_{n}\left\|x_{n}-x_{n-1}\right\| M } \\
& +\left(1-\gamma_{n}-\alpha_{n} \tau\right)\left(1+\theta_{n}\right) \sigma_{n}\left\|x_{n}-x_{n-1}\right\| M+2 \alpha_{n}\left\langle(v f-\rho F) p, x_{n+1}-p\right\rangle \\
\leq & {\left[1-\frac{\alpha_{n}(\tau-v \delta)}{2}\right]\left\|x_{n}-p\right\|^{2}+\sigma_{n}\left\|x_{n}-x_{n-1}\right\| M+2 \alpha_{n}\left\langle(v f-\rho F) p, x_{n+1}-p\right\rangle } \\
= & {\left[1-\frac{\alpha_{n}(\tau-v \delta)}{2}\right]\left\|x_{n}-p\right\|^{2}+\frac{\alpha_{n}(\tau-v \delta)}{2}\left[\frac{4\left\langle(v f-\rho F) p, x_{n+1}-p\right\rangle}{\tau-v \delta}+\frac{\sigma_{n}}{\alpha_{n}} \cdot \frac{\left\|x_{n}-x_{n-1}\right\| 2 M}{\tau-v \delta}\right] . }
\end{aligned}
$$

Step 4. One claims that $x_{n} \rightarrow x^{*} \in \Omega$, which is only a solution to the VIP (17). In fact, using the similar inference to that in Step 4 for the proof of Theorem 1, one derives the desired conclusion.

Example 1. We can get an example of $T$ satisfying the condition assumed in Theorems 1 and 2 . As a matter of fact, we put $H=\mathbf{R}$, whose inner product and induced norm are defined by $\langle a, b\rangle=a b$ and $\|\cdot\|=|\cdot|$ indicate, respectively. Let $T: H \rightarrow H$ be defined as $T x:=\sin \left(\frac{7}{8} x\right) \forall x \in H$. Then $T$ is a contraction with constant $\frac{7}{8}$, and hence a nonexpansive mapping. Thus, $T$ is an asymptotically nonexpansive mapping. As

$$
\left\|T^{n} x-T^{n} y\right\| \leq \frac{7}{8}\left\|T^{n-1} x-T^{n-1} y\right\| \leq \cdots \leq\left(\frac{7}{8}\right)^{n}\|x-y\| \quad \forall x, y \in H,
$$

we know that for any sequence $\left\{x_{n}\right\} \subset H$,

$$
\left\|T^{n+1} x_{n}-T^{n} x_{n}\right\| \leq\left(\frac{7}{8}\right)^{n-1}\left\|T^{2} x_{n}-T x_{n}\right\|=\left(\frac{7}{8}\right)^{n-1}\left\|\sin \left(\frac{7}{8} T x_{n}\right)-\sin \left(\frac{7}{8} x_{n}\right)\right\| \leq 2\left(\frac{7}{8}\right)^{n-1} \rightarrow 0
$$

as $n \rightarrow \infty$. That is, $T^{n} x_{n}-T^{n+1} x_{n} \rightarrow 0(n \rightarrow \infty)$.

Remark 3. Compared with the corresponding results in Bnouhachem et al. [2], Cai et al. [35], Kraikaew and Saejung [36], and Thong and Hieu [37,38], our results improve and extend them in what follows.

(i) The problem of obtaining a point of $\operatorname{VI}(C, A)$ in the work by the authors of [36] is extendable to the development of our problem of obtaining a point of $\cap_{i=0}^{N} \operatorname{Fix}\left(T_{i}\right) \cap \operatorname{VI}(C, A)$, where $T_{0}:=T$ is asymptotically nonexpansive and $\left\{T_{i}\right\}_{i=1}^{N}$ is a pool of nonexpansive maps. The Halpern subgradient method for solving the VIP in the work by the authors of [36] is extendable to the development of our mildly inertial subgradient algorithms 
with linesearch process for solving the VIP and CFPP.

(ii) The problem of obtaining a point of $\operatorname{VI}(C, A)$ in the work by the authors of [37] is extendable to the development of our problem of finding a point of $\cap_{i=0}^{N} \operatorname{Fix}\left(T_{i}\right) \cap \operatorname{VI}(C, A)$, where $T_{0}:=T$ is asymptotically nonexpansive and $\left\{T_{i}\right\}_{i=1}^{N}$ is a pool of nonexpansive maps. The inertial subgradient method with weak convergence for solving the VIP in the work by the authors of [37] is extendable to the development of our mildly inertial subgradient algorithms with linesearch process (which are convergent in norm) for solving the VIP and CFPP.

(iii) The problem of obtaining a point of $\operatorname{VI}(C, A) \cap \operatorname{Fix}(T)$ (where $A$ is monotone and $T$ is quasi-nonexpansive) in the work by the authors of [38] is extendable to the development of our problem of obtaining a point of $\cap_{i=0}^{N} \operatorname{Fix}\left(T_{i}\right) \cap \operatorname{VI}(C, A)$, where $T_{0}:=T$ is asymptotically nonexpansive and $\left\{T_{i}\right\}_{i=1}^{N}$ is a pool of nonexpansive maps. The inertial subgradient extragradient method with linesearch (which is weakly convergent) for solving the VIP and FPP in the work by the authors of [38] is extendable to the development of our mildly inertial subgradient algorithms with linesearch process (which are convergent in norm) for solving the VIP and CFPP. It is worth mentioning that the inertial subgradient method with linesearch process in the work by the authors of [38] combines the inertial subgradient approaches [37] with the Mann method.

(iv) The problem of obtaining a point in the common fixed-point set $\cap_{i=1}^{N} \operatorname{Fix}\left(T_{i}\right)$ of $N$ nonexpansive mappings $\left\{T_{i}\right\}_{i=1}^{N}$ in the work by the authors of [2], is extendable to the development of our problem of obtaining a point of $\cap_{i=0}^{N} \operatorname{Fix}\left(T_{i}\right) \cap \operatorname{VI}(C, A)$, where $T_{0}:=T$ is asymptotically nonexpansive and $\left\{T_{i}\right\}_{i=1}^{N}$ is a pool of nonexpansive maps. The iterative algorithm for hierarchical FPPs for finitely many nonexpansive mappings in the work by the authors of [2] (i.e., iterative scheme (3) in this paper), is extendable to the development of our mildly inertial subgradient algorithms with linesearch process for solving the VIP and CFPP. Meantime, the restrictions $\limsup _{n \rightarrow \infty} \gamma_{n}<1, \liminf _{n \rightarrow \infty} \gamma_{n}>0$ and $\lim _{n \rightarrow \infty}\left|\delta_{n-1}^{i}-\delta_{i, n}\right|=0$ for $i=1, \ldots, N$ imposed on (3), are dropped, where $0<\liminf _{n \rightarrow \infty} \gamma_{n}<\limsup _{n \rightarrow \infty} \gamma_{n}<1$ is weakened to the condition $0<\liminf _{n \rightarrow \infty} \gamma_{n} \leq \limsup _{n \rightarrow \infty} \gamma_{n}<1$.

(v) The problem of obtaining a point in the common solution set $\Omega$ of the VIPs for two inverse-strongly monotone mappings and the FPP of an asymptotically nonexpansive mapping in the work by the authors of [35], is extendable to the development of our problem of obtaining a point of $\cap_{i=0}^{N} \operatorname{Fix}\left(T_{i}\right) \cap \operatorname{VI}(C, A)$ where $T_{0}:=T$ is asymptotically nonexpansive and $\left\{T_{i}\right\}_{i=1}^{N}$ is a pool of nonexpansive maps. The viscosity implicit rule involving a modified extragradient method in the work by the authors of [35] (i.e., iterative scheme (4) in this paper), is extendable to the development of our mildly inertial subgradient algorithms with linesearch process for solving the VIP and CFPP. Moreover, the conditions $\sum_{n=1}^{\infty}\left|\alpha_{n+1}-\alpha_{n}\right|<\infty$ and $\sum_{n=1}^{\infty}\left\|T^{n+1} y_{n}-T^{n} y_{n}\right\|<\infty$ imposed on (4), are deleted where $\sum_{n=1}^{\infty}\left\|T^{n+1} y_{n}-T^{n} y_{n}\right\|<\infty$ is weakened to the assumption $\left\|T^{n+1} x_{n}-T^{n} x_{n}\right\| \rightarrow 0(n \rightarrow \infty)$.

\section{Applications}

In this section, our main theorems are used to deal with the VIP and CFPP in an illustrating example. The initial point $x_{0}=x_{1}$ is randomly chosen in $\mathbf{R}$. Take $v f(x)=F(x)=\frac{1}{2} x, \gamma=l=\mu=$ $\frac{1}{2}, \sigma_{n}=\alpha_{n}=\frac{1}{n+1}, \beta_{n}=\frac{1}{3}, \gamma_{n}=\frac{1}{2}, v=\frac{3}{4}, f=\frac{2}{3} I$ and $\rho=2$. Then, we know that $\alpha_{n}+\gamma_{n} \leq 1 \forall n \geq 1$, $v \delta=\kappa=\eta=\frac{1}{2}$, and

$$
\tau=1-\sqrt{1-\rho\left(2 \eta-\rho \kappa^{2}\right)}=1-\sqrt{1-2\left(2 \cdot \frac{1}{2}-2\left(\frac{1}{2}\right)^{2}\right)}=1 \in(0,1] .
$$

We first provide an example of a Lipschitzian, pseudomonotone operator $A$, asymptotically nonexpansive operator $T$, and nonexpansive operator $T_{1}$ with $\Omega=\operatorname{Fix}(T) \cap \operatorname{Fix}\left(T_{1}\right) \cap \operatorname{VI}(C, A) \neq \varnothing$. Let $C=[-1,3]$ and $H=\mathbf{R}$ with the inner product $\langle a, b\rangle=a b$ and induced norm $\|\cdot\|=|\cdot|$. Let $A, T, T_{1}, T_{1}^{n}: H \rightarrow H$ be defined as $A x:=\frac{1}{1+|\sin x|}-\frac{1}{1+|x|}, T x:=\frac{4}{5} \sin x, T x:=\sin x$ and 
$T_{1}^{n} x:=\frac{3}{8} x+\frac{5}{8} \sin x \forall x \in H, n \geq 1$. Then it is clear that $T_{1}$ is a nonexpansive mapping on $H$. Moreover, from Lemma 5 we know that $\operatorname{Fix}\left(T_{1}^{n}\right)=\operatorname{Fix}\left(T_{1}\right)=\{0\} \forall n \geq 1$. Now, we first show that $A$ is Lipschitzian, pseudomonotone operator with $L=2$. In fact, for all $x, y \in H$ we get

$$
\begin{aligned}
\|A x-A y\| & =\left|\frac{1}{1+\|\sin x\|}-\frac{1}{1+\|x\|}-\frac{1}{1+\|\sin y\|}+\frac{1}{1+\|y\|}\right| \\
& \leq\left|\frac{1}{1+\|\sin x\|}-\frac{1}{1+\|\sin y\|}\right|+\left|\frac{1}{1+\|x\|}-\frac{1}{1+\|y\|}\right| \\
& =\left|\frac{\|\sin y\|-\|\sin x\|}{(1+\|\sin x\|)(1+\|\sin y\|)}\right|+\left|\frac{\|y\|-\|x\|}{(1+\|x\|)(1+\|y\|)}\right| \\
& \leq\|\sin x-\sin y\|+\|x-y\| \\
& \leq 2\|x-y\| .
\end{aligned}
$$

This means that $A$ is Lipschitzian with $L=2$. We below claim that $A$ is pseudomonotone. For any given $x, y \in H$, it is clear that the relation holds:

$$
\langle A x, y-x\rangle=\left(\frac{1}{1+|\sin x|}-\frac{1}{1+|x|}\right)(y-x) \geq 0 \Rightarrow\langle A y, y-x\rangle=\left(\frac{1}{1+|\sin y|}-\frac{1}{1+|y|}\right)(y-x) \geq 0 .
$$

Furthermore, it is easy to see that $T$ is asymptotically nonexpansive with $\theta_{n}=\left(\frac{4}{5}\right)^{n} \forall n \geq 1$, such that $\left\|T^{n+1} x_{n}-T^{n} x_{n}\right\| \rightarrow 0$ as $n \rightarrow \infty$. Indeed, we observe that

$$
\left\|T^{n} x-T^{n} y\right\| \leq \frac{4}{5}\left\|T^{n-1} x-T^{n-1} y\right\| \leq \cdots \leq\left(\frac{4}{5}\right)^{n}\|x-y\| \leq\left(1+\theta_{n}\right)\|x-y\|,
$$

and

$$
\left\|T^{n+1} x_{n}-T^{n} x_{n}\right\| \leq\left(\frac{4}{5}\right)^{n-1}\left\|T^{2} x_{n}-T x_{n}\right\|=\left(\frac{4}{5}\right)^{n-1}\left\|\frac{4}{5} \sin \left(T x_{n}\right)-\frac{4}{5} \sin x_{n}\right\| \leq 2\left(\frac{4}{5}\right)^{n} \rightarrow 0(n \rightarrow \infty) .
$$

It is clear that $\operatorname{Fix}(T)=\{0\}$ and

$$
\lim _{n \rightarrow \infty} \frac{\theta_{n}}{\alpha_{n}}=\lim _{n \rightarrow \infty} \frac{(4 / 5)^{n}}{1 /(n+1)}=0
$$

Therefore, $\Omega=\operatorname{Fix}(T) \cap \operatorname{Fix}\left(T_{1}\right) \cap \operatorname{VI}(C, A)=\{0\} \neq \varnothing$. In this case, Algorithm 3 can be rewritten as follows,

$$
\left\{\begin{array}{l}
u_{n}=x_{n}+\frac{1}{n+1}\left(x_{n}-x_{n-1}\right), \\
y_{n}=P_{C}\left(u_{n}-\ell_{n} A u_{n}\right), \\
z_{n}=\frac{1}{3} x_{n}+\frac{2}{3} T_{1}^{n} P_{C_{n}}\left(u_{n}-\ell_{n} A y_{n}\right), \\
x_{n+1}=\frac{1}{n+1} \cdot \frac{1}{2} x_{n}+\frac{1}{2} x_{n}+\left(\frac{n}{n+1}-\frac{1}{2}\right) T^{n} z_{n} \quad \forall n \geq 1,
\end{array}\right.
$$

where for every $n \geq 1, C_{n}$ and $\ell_{n}$ are picked up as in Algorithm 3. Then, by Theorem 1, we know that $\left\{x_{n}\right\}$ converges to $0 \in \Omega=\operatorname{Fix}(T) \cap \operatorname{Fix}\left(T_{1}\right) \cap \operatorname{VI}(C, A)$ if and only if $\left|x_{n}-x_{n+1}\right|+\left|x_{n}-T_{1}^{n} x_{n}\right| \rightarrow 0$ as $n \rightarrow \infty$.

On the other hand, Algorithm 4 can be rewritten as follows,

$$
\left\{\begin{array}{l}
u_{n}=x_{n}+\frac{1}{n+1}\left(x_{n}-x_{n-1}\right), \\
y_{n}=P_{C}\left(u_{n}-\ell_{n} A u_{n}\right), \\
z_{n}=\frac{1}{3} x_{n}+\frac{2}{3} T_{1}^{n} P_{C_{n}}\left(u_{n}-\ell_{n} A y_{n}\right), \\
x_{n+1}=\frac{1}{n+1} \cdot \frac{1}{2} x_{n}+\frac{1}{2} u_{n}+\left(\frac{n}{n+1}-\frac{1}{2}\right) T^{n} z_{n} \quad \forall n \geq 1,
\end{array}\right.
$$

where for every $n \geq 1, C_{n}$ and $\ell_{n}$ are picked up as in Algorithm 4. Then, by Theorem 2, we know that $\left\{x_{n}\right\}$ converges to $0 \in \Omega=\operatorname{Fix}(T) \cap \operatorname{Fix}\left(T_{1}\right) \cap \operatorname{VI}(C, A)$ if and only if $\left|x_{n}-x_{n+1}\right|+\left|x_{n}-T_{1}^{n} x_{n}\right| \rightarrow 0$ as $n \rightarrow \infty$.

Author Contributions: These authors contributed equally to this work. 
Funding: The first author was partially supported by the Innovation Program of Shanghai Municipal Education Commission (15ZZ068), Ph.D. Program Foundation of Ministry of Education of China (20123127110002), and Program for Outstanding Academic Leaders in Shanghai City (15XD1503100).

Conflicts of Interest: The authors declare no conflicts of interest.

\section{References}

1. Ceng, L.C.; Petruşel, A.; Yao, J.C.; Yao, Y. Hybrid viscosity extragradient method for systems of variational inequalities, fixed points of nonexpansive mappings, zero points of accretive operators in Banach spaces. Fixed Point Theory 2018, 19, 487-501. [CrossRef]

2. Bnouhachem, A.; Ansari, Q.H.; Yao, J.C. An iterative algorithm for hierarchical fixed point problems for a finite family of nonexpansive mappings. Fixed Point Theory Appl. 2015, 2015, 111. [CrossRef]

3. Ceng, L.C.; Petruşel, A.; Yao, J.C.; Yao, Y. Systems of variational inequalities with hierarchical variational inequality constraints for Lipschitzian pseudocontractions. Fixed Point Theory 2019, 20, 113-133. [CrossRef]

4. Qin, X.; An, N.T. Smoothing algorithms for computing the projection onto a Minkowski sum of convex sets. Comput. Optim. Appl. 2019. [CrossRef]

5. Censor, Y.; Gibali, A.; Reich, S. The subgradient extragradient method for solving variational inequalities in Hilbert space. J. Optim. Theory Appl. 2011, 148, 318-335. [CrossRef] [PubMed]

6. Takahahsi, W.; Yao, J.C. The split common fixed point problem for two finite families of nonlinear mappings in Hilbert spaces. J. Nonlinear Convex Anal. 2019, 20, 173-195.

7. Cho, S.Y.; Li, W.; Kang, S.M. Convergence analysis of an iterative algorithm for monotone operators. J. Inequal. Appl. 2013, 2013, 199. [CrossRef]

8. Chang, S.S.; Wen, C.F.; Yao, J.C. Zero point problem of accretive operators in Banach spaces. Bull. Malays. Math. Sci. Soc. 2019, 42, 105-118. [CrossRef]

9. Nguyen, L.V.; Qin, X. Some results on strongly pseudomonotone quasi-variational inequalities. Set-Valued Var. Anal. 2019. [CrossRef]

10. Ceng, L.C.; Postolache, M.; Wen, C.F.; Yao, Y. Variational inequalities approaches to minimization problems with constraints of generalized mixed equilibria and variational inclusions. Mathematics 2019, 7, 270. [CrossRef]

11. Ceng, L.C.; Ansari, Q.H.; Yao, J.C. Some iterative methods for finding fixed points and for solving constrained convex minimization problems. Nonlinear Aanl. 2011, 74, 5286-5302. [CrossRef]

12. Chen, J.; Kobis, E.; Yao, J.C. Optimality conditions and duality for robust nonsmooth multiobjective optimization problems with constraints. J. Optim. Theory Appl. 2019, 181, 411-436. [CrossRef]

13. Liu, L.; Qin, X.; Agarwal, R.P. Iterative methods for fixed points and zero points of nonlinear mappings with applications. Optimization 2019. [CrossRef]

14. Cho, S.Y. Strong convergence analysis of a hybrid algorithm for nonlinear operators in a Banach space. J. Appl. Anal. Comput. 2018, 8, 19-31.

15. Ansari, Q.H.; Babu, F.; Yao, J.C. Regularization of proximal point algorithms in Hadamard manifolds. J. Fixed Point Theory Appl. 2019, 21, 25. [CrossRef]

16. Zhao, X. Linear regularity and linear convergence of projection-based methods for solving convex feasibility problems. Appl. Math. Optim. 2018, 78, 613-641. [CrossRef]

17. Cho, S.Y.; Kang, S.M. Approximation of common solutions of variational inequalities via strict pseudocontractions. Acta Math. Sci. 2012, 32, 1607-1618. [CrossRef]

18. Goebel, K.; Reich, S. Uniform Convexity, Hyperbolic Geometry, and Nonexpansive Mappings; Marcel Dekker: New York, NY, USA, 1984.

19. Chang, S.S.; Wen, C.F.; Yao, J.C. Common zero point for a finite family of inclusion problems of accretive mappings in Banach spaces. Optimization 2018, 67, 1183-1196. [CrossRef]

20. Ceng, L.C.; Ansari, Q.H.; Yao, J.C. An extragradient method for solving split feasibility and fixed point problems. Comput. Math. Appl. 2012, 64, 633-642. [CrossRef]

21. Ceng, L.C.; Liu, Z.; Yao, J.C.; Yao, Y. Optimal control of feedback control systems governed by systems of evolution hemivariational inequalities. Filomat 2018, 32, 5205-5220. [CrossRef]

22. Korpelevich, G.M. The extragradient method for finding saddle points and other problems. Ekonomikai Matematicheskie Metody 1976, 12, 747-756. 
23. Takahashi, W.; Wen, C.F.; Yao, J.C. The shrinking projection method for a finite family of demimetric mappings with variational inequality problems in a Hilbert space. Fixed Point Theory 2018, 19, 407-419. [CrossRef]

24. Vuong, P.T. On the weak convergence of the extragradient method for solving pseudo-monotone variational inequalities. J. Optim. Theory Appl. 2018, 176, 399-409. [CrossRef]

25. Vuong, P.T.; Shehu, Y. Convergence of an extragradient-type method for variational inequality with applications to optimal control problems. Numer. Algorithms 2019, 81, 269-291. [CrossRef]

26. Ceng, L.C.; Ansari, Q.H.; Wong, M.M.; Yao, J.C. Mann type hybrid extragradient method for variational inequalities, variational inclusions and fixed point problems. Fixed Point Theory 2012, 13, 403-422.

27. Ceng, L.C.; Wen, C.F. Systems of variational inequalities with hierarchical variational inequality constraints for asymptotically nonexpansive and pseudocontractive mappings. RACSAM 2019, 113, 2431-2447. [CrossRef]

28. Shehu, Y.; Dong, Q.; Jiang, D. Single projection method for pseudo-monotone variational inequality in Hilbert spaces. Optimization 2019, 68, 385-409. [CrossRef]

29. Shehu, Y.; Iyiola, O.S. Strong convergence result for monotone variational inequalities. Numer. Algorithms 2017, 76, 259-282. [CrossRef]

30. Ceng, L.C.; Hadjisavvas, N.; Wong, N.C. Strong convergence theorem by a hybrid extragradient-like approximation method for variational inequalities and fixed point problems. J. Glob. Optim. 2010, 46, 635-646. [CrossRef]

31. Ceng, L.C.; Teboulle, M.; Yao, J.C. Weak convergence of an iterative method for pseudomonotone variational inequalities and fixed-point problems. J. Optim. Theory Appl. 2010, 146, 19-31. [CrossRef]

32. Ceng, L.C.; Ansari, Q.H.; Wong, N.C.; Yao, J.C. An extragradient-like approximation method for variational inequalities and fixed point problems. Fixed Point Theory Appl. 2011, 2011, 22. [CrossRef]

33. Ceng, L.C.; Ansari, Q.H.; Yao, J.C. Relaxed extragradient iterative methods for variational inequalities. Appl. Math. Comput. 2011, 218, 1112-1123. [CrossRef]

34. Ceng, L.C.; Ansari, Q.H.; Schaible, S. Hybrid extragradient-like methods for generalized mixed equilibrium problems, systems of generalized equilibrium problems and optimization problems. J. Glob. Optim. 2012, 53, 69-96. [CrossRef]

35. Cai, G.; Shehu, Y.; Iyiola, O.S. Strong convergence results for variational inequalities and fixed point problems using modified viscosity implicit rules. Numer. Algorithms 2018, 77, 535-558. [CrossRef]

36. Kraikaew, R.; Saejung, S. Strong convergence of the Halpern subgradient extragradient method for solving variational inequalities in Hilbert spaces. J. Optim. Theory Appl. 2014, 163, 399-412. [CrossRef]

37. Thong, D.V.; Hieu, D.V. Inertial subgradient extragradient algorithms with line-search process for solving variational inequality problems and fixed point problems. Numer. Algorithms 2019, 80, 1283-1307. [CrossRef]

38. Thong, D.V.; Hieu, D.V. Modified subgradient extragradient method for variational inequality problems. Numer. Algorithms 2018, 79, 597-610. [CrossRef]

39. Cottle R.W.; Yao, J.C. Pseudo-monotone complementarity problems in Hilbert space. J. Optim. Theory Appl. 1992, 75, 281-295. [CrossRef]

40. Xue, Z.; Zhou, H.; Cho, Y.J. Iterative solutions of nonlinear equations for $\mathrm{m}$-accretive operators in Banach spaces. J. Nonlinear Convex Anal. 2000, 1, 313-320.

41. Zhou, H. Convergence theorems of fixed points for $\kappa$-strict pseudo-contractions in Hilbert spaces. Nonlinear Anal. 2008, 69, 456-462. [CrossRef]

42. Yamada, I. The hybrid steepest-descent method for variational inequalities problems over the intersection of the fixed point sets of nonexpansive mappings, In Inherently Parallel Algorithms in Feasibility and Optimization and Their Applications; Butnariu, D., Censor, Y., Reich, S., Eds.; North-Holland: Amsterdam, The Netherlands, 2001; pp. 473-504.

43. Goebel, K.; Kirk, W.A. Topics in Metric Fixed Point Theory (Cambridge Studies in Advanced Mathematics); Cambridge University Press: Cambridge, UK, 1990; Volume 28.

(C) 2019 by the authors. Licensee MDPI, Basel, Switzerland. This article is an open access article distributed under the terms and conditions of the Creative Commons Attribution (CC BY) license (http:// creativecommons.org/licenses/by/4.0/). 